\title{
Snap behaviour in the upheaval buckling of subsea pipelines under topographic step
}

\section{imperfection}

\author{
Zhenkui Wang, ${ }^{\mathrm{a}, \mathrm{b}}$, G.H.M. van der Heijden ${ }^{\mathrm{b}, *}$, \\ a State Key Laboratory of Hydraulic Engineering Simulation and Safety, Tianjin University, Tianjin 300072, China \\ ${ }^{\mathrm{b}}$ Department of Civil, Environmental and Geomatic Engineering, University College London, London WC1E 6BT, UK
}

Corresponding author: G.H.M. van der Heijden, g.heijden@ucl.ac.uk

\begin{abstract}
Pipelines exposed to high-temperature and high-pressure (HTHP) with a topographic step imperfection are susceptible to the phenomenon of upheaval buckling potentially leading to a hazard for the structural integrity of the pipeline. To analyse this problem we derive analytical upheaval buckling solutions and obtain the locations of maximum displacement and maximum axial compressive stress. We also analyse the typical post-buckling behaviour and its dependence on step height, axial soil resistance and wall thickness. The difference in behaviour between a pipeline with step imperfection and one with a symmetric prop imperfection is discussed. Our results show that a pipeline with a step imperfection is more prone to upheaval buckling than a perfect pipeline. For sufficiently small step heights the pipeline may suffer a snap-back instability under decreasing thermal loading, raising the possibility of hysteretic snap behaviour under cyclic thermal loading (for instance caused by periodic start-ups and shut-downs). The snap-back buckling disappears for large enough step height and the minimum critical temperature difference decreases with increasing step height and wall thickness or with decreasing axial soil resistance. The maximum compressive stress decreases with increasing step height and axial soil resistance or with decreasing wall thickness. A pipeline with step imperfection is safer than one with a symmetric prop imperfection.
\end{abstract}

Keywords: Subsea pipelines; Upheaval buckling; Step imperfection.

\section{Introduction}

Submarine pipelines are widely used for transporting offshore oil and gas in high-temperature and high-pressure (HTHP) conditions to prevent solidification of the wax fraction in deep water [1]. These pipelines are generally laid on the seabed untrenched at ambient temperatures and are subject to heating and pressurisation during operation. Both the rise in temperature and in internal pressure may lead to longitudinal expansion of the pipeline. This expansion is restrained by the pipe end devices and the seabed, which can lead to the accumulation of effective axial compressive force in the pipe wall [2,3]. Due to the large effective axial force, lateral or upheaval buckling may be triggered. When a pipeline is laid on an uneven seabed, such as a seabed with topographic step imperfection, upheaval buckling (i.e., lift-off of the pipe on the higher section) may take place once the axial compressive force induced by thermal expansion and pressure increase is larger than the critical axial compressive force. Upheaval buckling is a structural response to a high axial compressive force and not a failure mode as such [4]. However, upheaval buckling may induce failure modes such as local buckling, fracture, fatigue or excessive displacement. Thus, it is important to investigate upheaval buckling behaviour of subsea pipelines laid on the seabed with a topographic step imperfection.

Studies on lateral and upheaval buckling of subsea pipelines have been carried out by many researchers through analytical and finite-element modelling. Hobbs [5, 6] may have been the first one to study lateral buckling of subsea pipelines based on the study of lateral buckling of railroad tracks by Kerr [7]. In their work, four classical lateral buckling modes were proposed and formulas for critical axial loads, buckling displacement amplitude and bending moment were presented. Then, based on Hobbs' analysis, Taylor and Gan derived an analytical solution to lateral and upheaval buckling for subsea pipelines with the consideration of a deformation-dependent resistance force model $[8,9]$. Furthermore, small-scale model tests were conducted to understand the mechanism of upheaval buckling of buried pipelines [10,11]. Four types of numerical simulation models were proposed by Liu [12] based on the finite-element method to simulate lateral buckling of pipelines under temperature difference. A novel way to introduce initial imperfection into the finite-element model was presented. More recently, analytical 
solutions were derived by Wang et al. through analytical method for controlled lateral buckling behaviour based on classic mode three [13] and the influence of a distributed buoyancy section or a sleeper on the lateral buckling behaviour based on mode one $[14,15]$ and mode three $[16,17]$ for unburied subsea pipelines. They found that post-buckling behaviour was affected significantly by the feed-in length. Lateral buckling was found to occur more easily than upheaval buckling and the maximum stress of the laterally buckled pipeline was found to decrease with a distributed buoyancy section or a sleeper. Furthermore, localised lateral buckling of partially embedded subsea pipelines and localised upheaval buckling of buried pipelines with nonlinear soil resistance were studied by Zeng and Duan [18] and Wang and co-workers [19, 20] without the assumption of lateral or upheaval deflection. In these studies, only ideal straight subsea pipelines were taken into consideration. No geometric initial imperfections, especially step imperfection, were included. However, in practice, step imperfections due to uneven topographic seabed may exist.

As to geometric initial imperfections, many previous researchers have focused on the influence of initial imperfection on upheaval and lateral buckling behaviour through analytical methods. Initial imperfections were incorporated into the analytical models by Taylor and co-workers [21, 22] for lateral and upheaval buckling of submarine pipelines. Furthermore, upheaval creep behaviour due to initial imperfections was investigated by Pedersen and Jensen presented [23] for pipelines subjected to time-varying temperature loadings. The asymmetric effects of prop imperfections on the upheaval buckling of pipelines were studied by Ballet and Hobbs [24]. They found that an asymmetric mode of buckling may occur for pipelines with prop imperfections. Furthermore, a simplified analytical model was proposed for upheaval thermal buckling of pipelines with initially imperfect geometry by Croll [25]. Simple and closed-form solutions suitable to design were derived for both initial lift-off and the maximum upheaval buckling loads through this analytical model. Karampour and co-workers investigated the interaction between upheaval or lateral buckling [26] and propagation buckling of subsea pipelines [2, 27, 28] with the consideration of a single imperfection. The collapse of the pipe-wall due to the interaction between lateral buckling and external pressure was studied and buckle interaction envelopes were developed. An analytical model was presented by Shi et al. [29] to investigate the effect of seabed resistance on upheaval buckling of pipelines rested on a plastic soft seabed with a prop imperfection. The analytical solution of higher-order lateral buckling was derived by Hong et al. [30] for submarine pipelines with a single arch symmetric initial imperfection through the energy method. The analytical solution was compared with finite-element results. Wang et al. [31] presented a perturbation analysis for upheaval buckling of imperfect buried pipelines based on nonlinear pipe-soil interaction.

In more recent years, the finite-element method has been widely used in the investigation of thermal buckling for geometrically imperfect pipelines. Thermal upheaval buckling behaviour of buried pipelines with different amplitudes of initial geometric imperfection was studied by Liu et al. [32] through finite-element modelling with a nonlinear soil resistance model incorporated. They found that the critical temperature difference increases with the buried depth and decreases with the amplitude of the initial imperfection. Wang et al. [33] investigated the upheaval buckling behaviour and critical axial compressive force of pipe-in-pipe systems with full-contact imperfections through finite-element computations. Based on dimensional analysis and numerical results, they proposed two empirical formulas for two typical imperfection profiles to calculate the critical axial compressive force of pipe-in-pipe systems. Finite-element models were used by Wang et al. [34] to study the lateral buckling behaviour of pipelines with imperfection and sleeper. The effect of seabed undulation and initial stress of the pipeline on the critical axial compressive force of upheaval buckling was investigated by $\mathrm{Xu}$ and Lin [35] by using numerical tools developed with the Vector Form Intrinsic Finite-Element (VFIFE) method. Zhang et al. [36, 37] studied upheaval and lateral buckling behaviour of submarine pipelines with different initial imperfection shapes. A characteristic parameter of the initial imperfection shape was defined in their analysis, which had a great influence on the critical axial compressive force. They found that the location of occurrence of upheaval and lateral buckling was controlled by the minimum curvature value of the initial imperfection shape. A method to determine the model length of pipeline for the numerical simulation of pipe buckling was proposed by Liu and Li [38] through analytical and finite-element approaches. All these researches focused on symmetric geometric imperfections, rather than asymmetric ones, such as a step imperfection.

As for step buckling, Hunt and Blackmore [39] discussed several kinds of solutions of upheaval buckling to investigate the 
effect of asymmetric bed imperfections, such as step imperfections. However, temperature difference and axial soil resistance were not considered in this research. So the purpose of this paper is to study analytically the influence of an asymmetric topographic imperfection on pipeline thermal buckling behaviour. First, analytical solutions of upheaval buckling for subsea pipelines with topographic step imperfection under axial compressive force induced by temperature difference and internal pressure are derived. Then, the locations of maximum displacement and three extrema of axial compressive stress are obtained and the maximum axial compressive stress is determined. After that, the typical post-buckling behaviour is presented. In addition to the possibility of upheaval buckling through a snap-through instability this also highlights what we call a snapback instability from the buckled to the unbuckled state at a critical temperature difference. A detailed parametric analysis is carried out to determine the influence of step height, axial soil resistance and wall thickness on this snap behaviour as well as on maximum pipe stress. Finally, the upheaval buckling behaviour triggered by a step imperfection is compared to that triggered by a symmetric prop imperfection.

\section{Analytical solution}

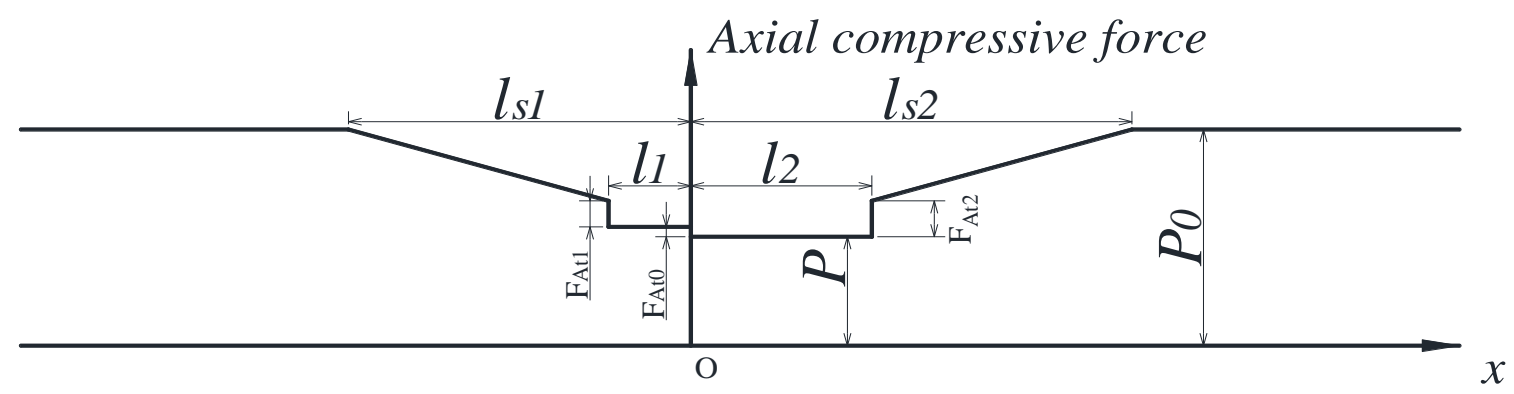

Fig. 1 Axial compressive force distribution.

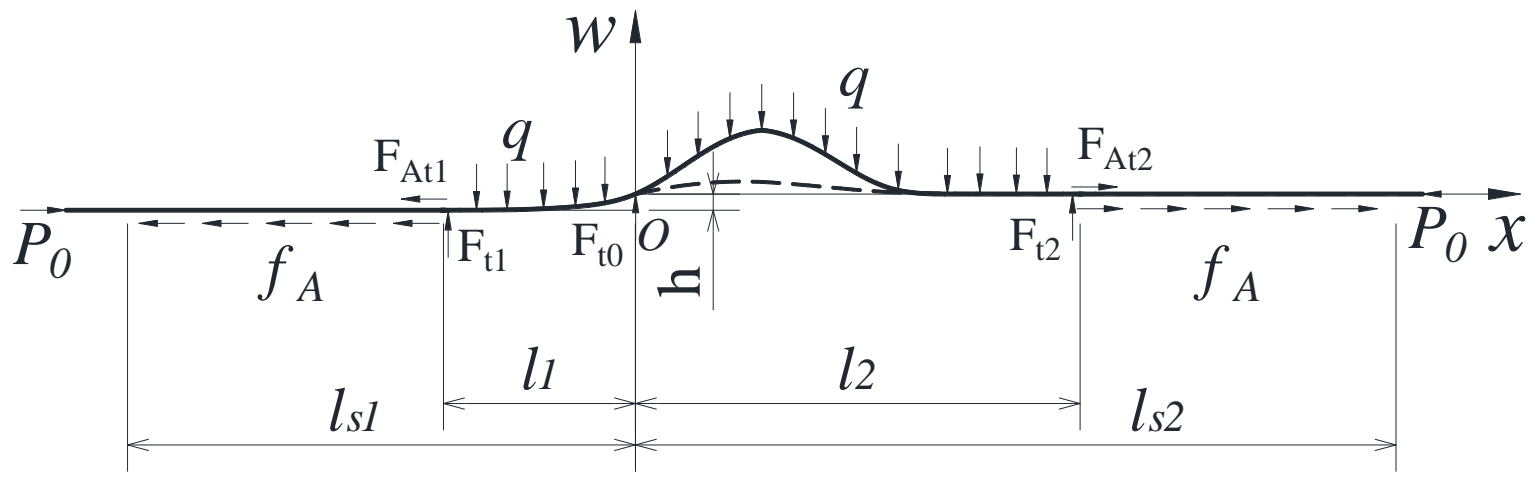

Fig. 2 Pipeline configuration and load distribution.

Pipelines tend to expand longitudinally to relieve the axial compressive forces created by internal pressure and temperature. This expansion is opposed by the axial soil resistance between the pipeline and the seabed, so that axial compressive forces build up along the length of the pipeline. In the process of thermal buckling, a small central segment of pipe will mobilise vertically (see Fig. 2). As additional pipe required for vertical deformation feeds into the buckle, the axial compressive force in the pipe drops. If the soil axial resistance is constant, say $f_{A}$, then the compressive force will increase linearly with the distance from the nearest touchdown point $x=-l_{1}$ or $x=l_{2}$ between pipeline and seabed. The segment $-l_{1} \leq x \leq l_{2}$ is called the buckle region. At some points $x=-l_{s 1}$ and $x=l_{s 2}$ the axial thermal expansion of the pipeline is sufficient to satisfy the requirement of additional length introduced by the vertical displacement. At these points the axial displacement is therefore zero. They are called virtual anchor points and bound what is called the feed-in region. Fig. 1 shows this feed-in region, of length $l_{s 1}+l_{s 2}$, within the larger immobilised section of the pipeline together with the typical axial compressive force distribution. $P_{0}$ is the axial compressive force at the virtual anchor points. Fig. 2 illustrates the configuration and load 
distribution of upheaval buckling for pipelines with a step imperfection. The dashed curve represents the initial deflection of the pipeline under its own weight (the deflection is exaggerated for easy visibility), while the solid curve represents the buckle shape of the pipeline under temperature difference. In the analytical formulation presented in this section the pipeline is modelled using linear beam-column theory valid for small deflections.

\subsection{Analytical solution of the initial configuration}

Consider a pipeline, perfectly straight in its unstressed state, resting upon a rigid seabed $[11,25]$ with topographic step imperfection. Before the operating phase, no axial compressive force exists along the pipeline due to the absence of temperature difference and internal pressure. Under its own submerged weight, the pipeline will adopt a deformed equilibrium state in the vertical plane as shown in Fig. 2. Thus, the governing equations for the initial configuration of the pipeline without axial compressive force are

$$
\left\{\begin{array}{lc}
E I \frac{\mathrm{d}^{4} v_{1}}{d x^{4}}=-q & \left(-l_{1} \leq x \leq 0\right) \\
E I \frac{\mathrm{d}^{4} v_{2}}{d x^{4}}=-q & \left(0 \leq x \leq l_{2}\right)
\end{array}\right.
$$

where $v_{1}$ and $v_{2}$ are the vertical deflections, $q$ is the submerged weight per unit length of the pipeline, $E$ is the elastic modulus, $I$ is the second moment of area of the cross-section, and $l_{1}$ and $l_{2}$ are the lengths of the deflected pipeline. The general solutions of Eq. (1) are

$$
\left\{\begin{array}{l}
v_{1}(x)=-\frac{q}{24 E I} x^{4}+A_{1} x^{3}+A_{2} x^{2}+A_{3} x+A_{4} \\
v_{2}(x)=-\frac{q}{24 E I} x^{4}+A_{5} x^{3}+A_{6} x^{2}+A_{7} x+A_{8}
\end{array}\right.
$$

where $A_{1}, \ldots, A_{8}$ are constant coefficients.

Let the step height be $h$, so that the displacement at $x=-l_{1}$ is $-h$, while the slope and moment at $x=-l_{1}$ are zero. Also, the displacement, slope and moment at $x=l_{2}$ must all be zero. So the boundary conditions can be written as

$$
\left\{\begin{array}{c}
v_{1}\left(-l_{1}\right)=-h \\
\frac{d v_{1}}{d x}\left(-l_{1}\right)=0 \\
\frac{d^{2} v_{1}}{d x^{2}}\left(-l_{1}\right)=0 \\
v_{2}\left(l_{2}\right)=0 \\
\frac{d v_{2}}{d x}\left(l_{2}\right)=0 \\
\frac{d^{2} v_{2}}{d x^{2}}\left(l_{2}\right)=0
\end{array}\right.
$$

In addition, the displacement, slope and bending moment must be continuous at the step, while the vertical deflection at the step must be zero. Thus, the following matching conditions must be satisfied

$$
\left\{\begin{array}{c}
v_{1}(0)=0 \\
v_{2}(0)=0 \\
\frac{d v_{1}}{d x}(0)=\frac{d v_{2}}{d x}(0) \\
\frac{d^{2} v_{1}}{d x^{2}}(0)=\frac{d^{2} v_{2}}{d x^{2}}(0)
\end{array}\right.
$$

Thus we have six boundary conditions in Eq. (3) and four matching conditions in Eq. (4). By using eight of them, combined with Eq. (2), the eight coefficients, $A_{1}, \ldots, A_{8}$, can be expressed as functions of $l_{1}$ and $l_{2}$ (see the Appendix). The remaining two boundary conditions can then be expressed as

$$
\left\{\begin{array}{l}
v_{2}\left(l_{2}\right)=\frac{l_{2}\left(24 E I h\left(3 l_{1}+2 l_{2}\right)+l_{1}{ }^{2} q\left(-l_{1}^{3}-2 l_{1}^{2} l_{2}+l_{2}^{3}\right)\right)}{24 E I l_{1}^{2}}=0 \\
\frac{d v_{2}}{d x}\left(l_{2}\right)=\frac{\left(l_{1}+l_{2}\right)\left(72 E I h-l_{1}^{2} q\left(l_{1}^{2}+2 l_{1} l_{2}-2 l_{2}^{2}\right)\right)}{24 E I l_{1}^{2}}=0
\end{array}\right.
$$

and can be used to calculate $l_{1}$ and $l_{2}$. Once the constant coefficients $A_{1}, \ldots, A_{8}$ are known, the vertical deflections $v_{1}$ and $v_{2}$ can be obtained. An example of such an initial configuration is shown in Fig. 2.

The geometric shortening $u_{20}$, required for the additional length introduced by the vertical displacement, can be calculated 
through

$$
u_{20}=\frac{1}{2} \int_{-l_{1}}^{0}\left(\frac{\mathrm{d} v_{1}}{\mathrm{~d} x}\right)^{2} d x+\frac{1}{2} \int_{0}^{l_{2}}\left(\frac{\mathrm{d} v_{2}}{\mathrm{~d} x}\right)^{2} d x
$$

\subsection{Analytical solution for the buckled pipeline}

The straight pipeline is assumed to be installed on the seabed at ambient temperature and in stress-free conditions. Let the temperature of the pipeline be raised by an amount $T_{0}$, composed of the initial temperature difference and the equivalent temperature difference generated by internal pressure. If the pipe were free to expand it would do so, with a longitudinal strain $\alpha T_{0}$ where $\alpha$ is the coefficient of thermal expansion of the material. However, the expansion is opposed by the axial resistance between the pipe and the seabed, so an axial compressive force, $P_{0}=E A \alpha T_{0}$, builds up along the length of the pipeline, assuming the pipeline remains straight. Here $A$ is the cross-sectional area of the pipeline.

When the axial compressive force $P_{0}$ is large enough the pipeline tends to buckle to relieve the stress created by temperature difference and internal pressure. In the following analysis we assume that the buckled pipeline always rests on the step at $x=0$, which is expected to be the case for sufficiently long pipelines [39]. With reference to Fig. 1, the axial compressive force distribution $\bar{P}(x)$ during the buckling stage can be expressed as

$$
\bar{P}(x)=\left\{\begin{array}{cc}
P+F_{A t 0}+F_{A t 1}-f_{A}\left(x+l_{1}\right) & \left(-l_{s 1} \leq x<-l_{1}\right) \\
P+F_{A t 0} & \left(-l_{1}<x<0\right) \\
P & \left(0<x<l_{2}\right) \\
P+F_{A t 2}+f_{A}\left(x-l_{2}\right) & \left(l_{2}<x \leq l_{s 2}\right)
\end{array}\right.
$$

where $F_{A t 0}=\mu_{A} F_{t 0}, F_{A t 1}=\mu_{A} F_{t 1}$ and $F_{A t 2}=\mu_{A} F_{t 2}$ are the concentrated axial friction forces induced by the concentrated contact forces $F_{t 0}, F_{t 1}$ and $F_{t 2}$ at the touchdown points $x=0, x=-l_{1}$ and $x=l_{2}$, respectively. $\mu_{A}$ is the axial friction coefficient between pipeline and seabed. $l_{s 1}$ and $l_{s 2}$ are the feed-in lengths. The total feed-in length is $l_{s t}=l_{s 1}+l_{s 2} . P$ is the axial compressive force within the buckle region $0 \leq x \leq l_{2}$.

Therefore, the axial compressive forces at the anchor points $x=l_{s 1}$ and $x=l_{s 2}$ are

$$
\left\{\begin{array}{c}
\bar{P}\left(-l_{s 1}\right)=P_{0}=P+F_{A t 0}+F_{A t 1}-f_{A}\left(-l_{s 1}+l_{1}\right) \\
\bar{P}\left(l_{s 2}\right)=P_{0}=P+F_{A t 2}+f_{A}\left(l_{s 2}-l_{2}\right)
\end{array}\right.
$$

According to Eq. (8), the relationship between feed-in length $l_{s 1}$ and $l_{s 2}$ can be obtained as

$$
l_{s 1}=l_{s 2}+l_{1}-l_{2}+\left(F_{A t 2}-F_{A t 0}-F_{A t 1}\right) / f_{A}
$$

The equilibrium equations governing the buckled pipeline with axial compressive force are

$$
\left\{\begin{array}{lc}
E I \frac{d^{4} w_{1}}{d x^{4}}+P \frac{d^{2} w_{1}}{d x^{2}}=-q & \left(-l_{1} \leq x \leq 0\right) \\
E I \frac{d^{4} w_{2}}{d x^{4}}+P \frac{d^{2} w_{2}}{d x^{2}}=-q & \left(0 \leq x \leq l_{2}\right)
\end{array}\right.
$$

Here, for the purpose of determining the deflections $w_{1}, w_{2}$, we have made the simplifying assumption that, despite Eq. (7), the axial compressive force within the buckle region $-l_{1} \leq x \leq 0$ is equal to the force $P$, thereby ignoring the concentrated axial friction force $F_{A t 0}$. This is justified because $F_{A t 0}$ is much smaller than the axial compressive force $P$. (Quantitative justification for the assumption is given in Fig. 4 below.)

Now let

$$
\lambda^{2}=\frac{P}{E I}
$$

The general solutions of Eq. (10) can then be written as

$$
\left\{\begin{array}{lr}
w_{1}(x)=B_{1} \cos \lambda x+B_{2} \sin \lambda x+B_{3} x+B_{4}-\frac{q}{2 \lambda^{2} E I} x^{2} & \left(-l_{1} \leq x \leq 0\right) \\
w_{2}(x)=B_{5} \cos \lambda x+B_{6} \sin \lambda x+B_{7} x+B_{8}-\frac{q}{2 \lambda^{2} E I} x^{2} & \left(0 \leq x \leq l_{2}\right)
\end{array}\right.
$$

The boundary conditions and continuity conditions are the same as those listed in Eq. (3) and Eq. (4), which can be written as 


$$
\begin{gathered}
\left\{\begin{array}{c}
w_{1}\left(-l_{1}\right)=-h \\
\frac{d w_{1}}{d x}\left(-l_{1}\right)=0 \\
\frac{d^{2} w_{1}}{d x^{2}}\left(-l_{1}\right)=0 \\
w_{2}\left(l_{2}\right)=0 \\
\frac{d w_{2}}{d x}\left(l_{2}\right)=0 \\
\frac{d^{2} w_{2}}{d x^{2}}\left(l_{2}\right)=0
\end{array}\right. \\
\left\{\begin{array}{c}
w_{1}(0)=0 \\
w_{2}(0)=0 \\
\frac{d w_{1}}{d x}(0)=\frac{d w_{2}}{d x}(0) \\
\frac{d^{2} w_{1}}{d x^{2}}(0)=\frac{d^{2} w_{2}}{d x^{2}}(0)
\end{array}\right.
\end{gathered}
$$

Once $w_{1}$ and $w_{2}$ have been found the point contact force $F_{t 0}$ between the pipeline and the seabed at the step can be calculated as

$$
F_{t 0}=E I \frac{d^{3} w_{2}}{d x^{3}}(0)-E I \frac{d^{3} w_{1}}{d x^{3}}(0)
$$

and the concentrated contact forces $F_{t 1}$ and $F_{t 2}$ at $x=-l_{1}$ and $x=l_{2}$ can be obtained through

$$
\left\{\begin{array}{l}
F_{t 1}=E I \frac{d^{3} w_{1}}{d x^{3}}\left(-l_{1}\right) \\
F_{t 2}=-E I \frac{d^{3} w_{2}}{d x^{3}}\left(l_{2}\right)
\end{array}\right.
$$

We now use compatibility between axial and vertical deformation in the feed-in zone $-l_{s 1} \leq x \leq l_{s 2}$ to derive a relationship between the axial compressive force $P$ and the temperature difference $T_{0}$ that causes upheaval buckling. Compatibility can be expressed as

$$
u_{1}=u_{2}
$$

where $u_{1}$ is the length of axial thermal expansion within the pipeline section $-l_{s 1} \leq x \leq l_{s 2}$ due to high pressure and high temperature. $u_{2}$ is the geometric shortening, which allows for the additional length introduced by the vertical displacement. Eq. (17) simply states that the extra length of pipe in the buckle must come from axial thermal expansion of the mobilised segment of pipe.

The length of axial thermal expansion $u_{1}$ can be calculated by

$$
u_{1}=\int_{-l_{s 1}}^{l_{s 2}} \frac{\Delta \bar{P}(x)}{E A} d x
$$

where $\Delta \bar{P}(x)=P_{0}-\bar{P}(x)$ is the amount of reduction of axial compressive force along the pipeline after the pipeline buckles. Meanwhile, for $u_{2}$ we have

$$
u_{2}=\frac{1}{2} \int_{-l_{1}}^{0}\left(\frac{d w_{1}}{d x}\right)^{2} d x+\frac{1}{2} \int_{0}^{l_{2}}\left(\frac{d w_{2}}{d x}\right)^{2} d x-u_{20}
$$

The bending moment $M$ along the buckled pipeline can be obtained by

$$
M=E I \frac{d^{2} w}{d x^{2}}
$$

where $w$ stands for either $w_{1}$ or $w_{2}$. Thus, the corresponding bending stress $\sigma_{M}$ along the buckled pipeline is

$$
\sigma_{M}=\frac{M D}{2 I}
$$

where $\mathrm{D}$ is the external diameter of the pipe.

The maximum axial compressive stress $\sigma_{m}$ along the pipeline can be obtained from the following expression

$$
\sigma_{m}=\sigma_{P}+\left|\sigma_{M m}\right|
$$

where the stresses $\sigma_{P}$ and $\sigma_{M m}$, induced by axial compressive force $P$ and maximum bending moment $M_{m}$ respectively, 
can be obtained by

$$
\left\{\begin{array}{c}
\sigma_{P}=\frac{P}{A} \\
\sigma_{M m}=\frac{M_{m} D}{2 I}
\end{array}\right.
$$

\section{Analytical results}

\subsection{The values of $\lambda l_{1}$ and $\lambda l_{2}$}

The solution for the pipeline deflection $w_{1}, w_{2}$ can be presented in semi-explicit form by using eight of the Eq. (13) and Eq. (14) to express the coefficients $B_{i}$ in terms of $\lambda, l_{1}$ and $l_{2}$ and all the parameters of the problem (see the Appendix). Two remaining transcendental equations then need to be solved to obtain the values of $\lambda l_{1}$ and $\lambda l_{2}$ given $\lambda$. For pipelines without step imperfection, i.e., $h=0$, the values of $\lambda l_{1}$ and $\lambda l_{2}$ are 0 and 8.9868, respectively, for all values of $T_{0}$ (see the solid lines in Fig. 3). For pipelines with step imperfection $(h>0)$, the values of $\lambda l_{1}$ and $\lambda l_{2}$ change with the total temperature difference $T_{0}$ and the step height $h$. The relationships between $\lambda l_{1}, \lambda l_{2}$ and $T_{0}$ at various step heights are given graphically in Fig. 3. We see that a fold exists for a small step height, such as $h=0.6 D$ or $1.5 D$ (indicating multiple solutions), but that for a large step height $(h=3.0 D)$ the fold disappears.

Fig. 4 presents values of $F_{A t 0} / P$ for various step heights. All values of $F_{A t 0} / P$ are seen to be smaller than 0.015 . This confirms therefore that the approximation made in writing down the governing equations (Eq. (10)) is justified.

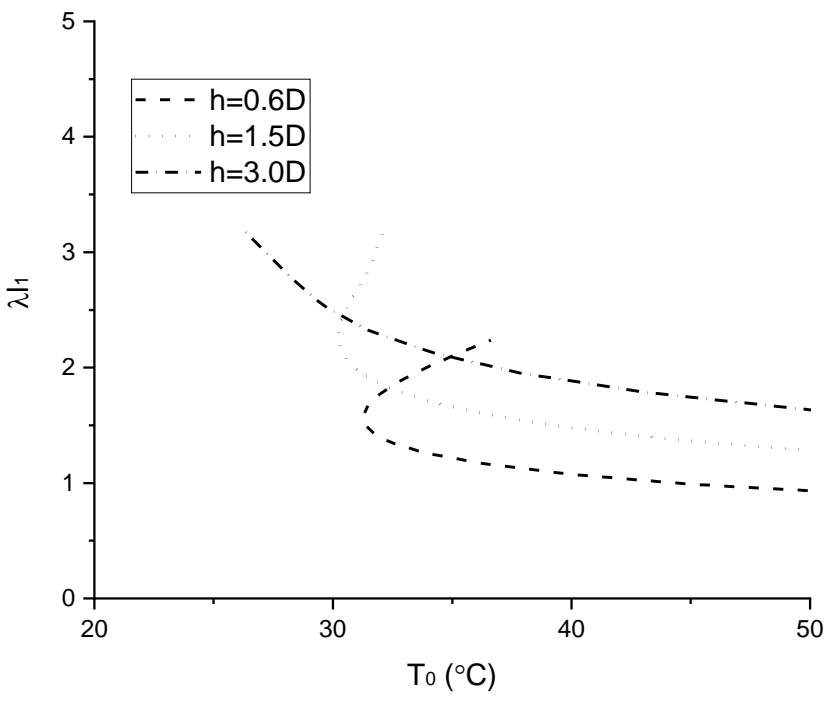

(a)

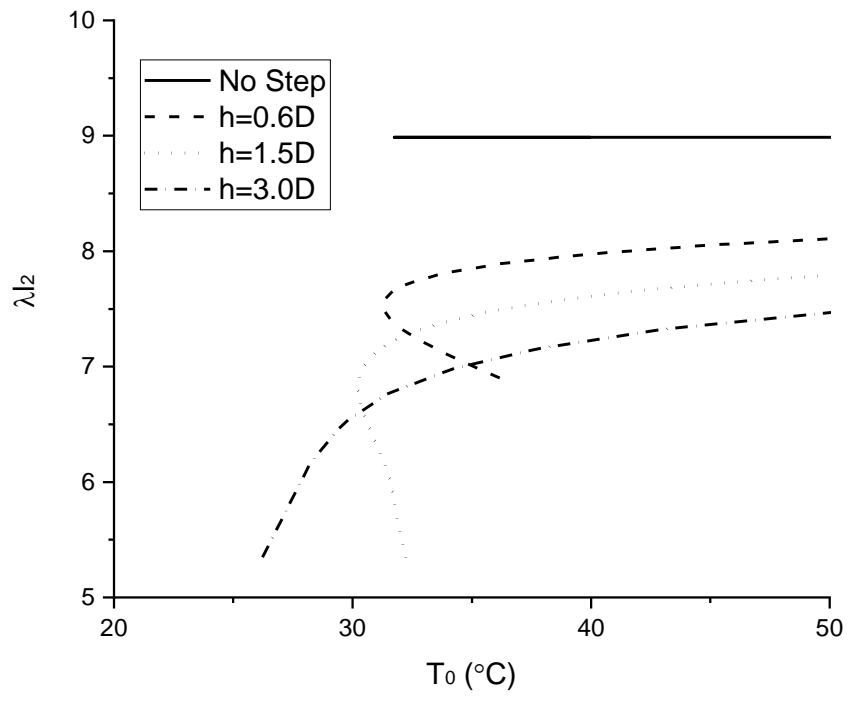

(b)

Fig. 3 The values of $\lambda l_{1}$ and $\lambda l_{2}$. (a) Relationship between $\lambda l_{1}$ and $T_{0}$. (b) Relationship between $\lambda l_{2}$ and $T_{0}$.

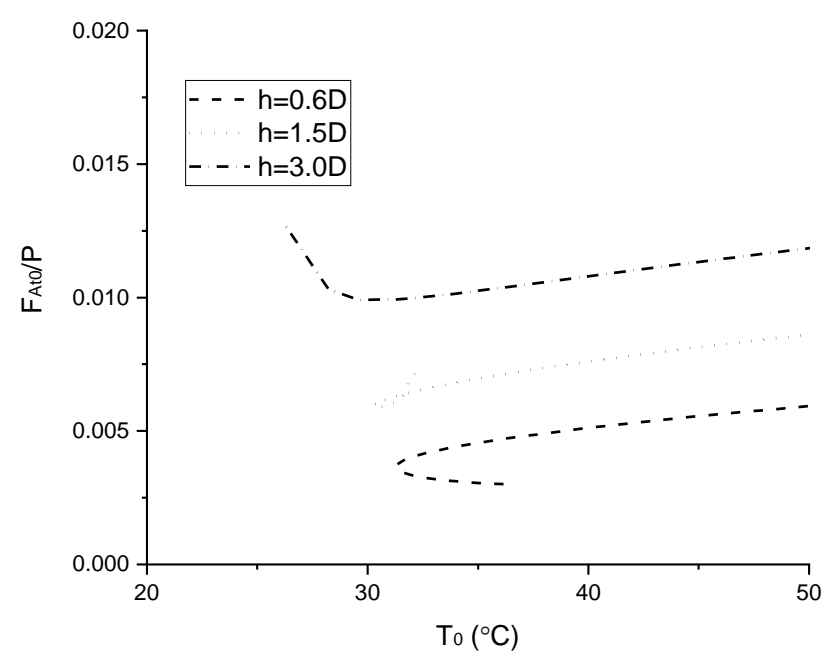

Fig. 4 Typical values of $F_{A t 0} / P$. 


\subsection{The locations of $w_{m}$ and $\sigma_{m}$}

The deformed shapes and the corresponding bending stresses $\sigma_{M}$ along the buckled pipeline under different temperature differences are presented in Fig. 5. Note that a smaller lobe is formed beneath the step, while a larger lobe exists above the step for each buckled shape, as shown in Fig. 5(a). The small lobe beneath the step is barely affected by the temperature difference, while the large lobe above the step enlarges significantly with increasing temperature difference. Only one maximum displacement, denoted as $w_{m}$, is seen to exist above the step. It increases and the location of it moves away from the step with increasing temperature difference.

In Fig. 5(b), we see that there exist three extreme values of bending stress along the buckled pipeline, whose locations move to or away from the step for different temperature differences. The axial compressive stress is composed of bending stress and stress induced by axial compressive force. The axial compressive force is constant within the buckled region, so the locations of extreme values of the bending stress are the same as the locations of extreme values of the axial compressive stress. The three extreme values of the axial compressive stress and their locations are denoted as $\sigma_{m 1}, \sigma_{m 2}, \sigma_{m 3}$ and $x_{\sigma m 1}$, $x_{\sigma m 2}, x_{\sigma m 3}$ from left to right, respectively, as showed in Fig. 5(b).

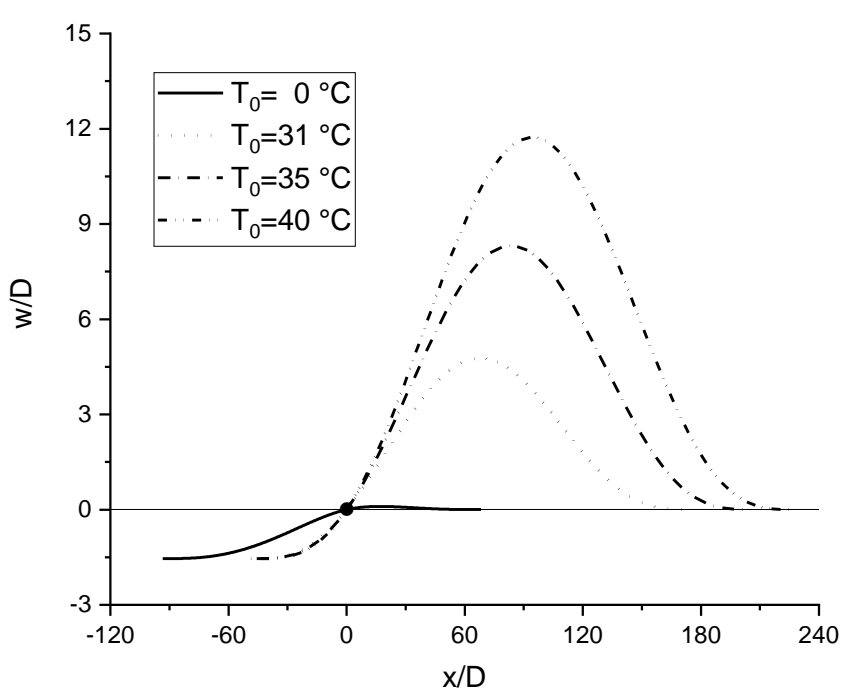

(a)

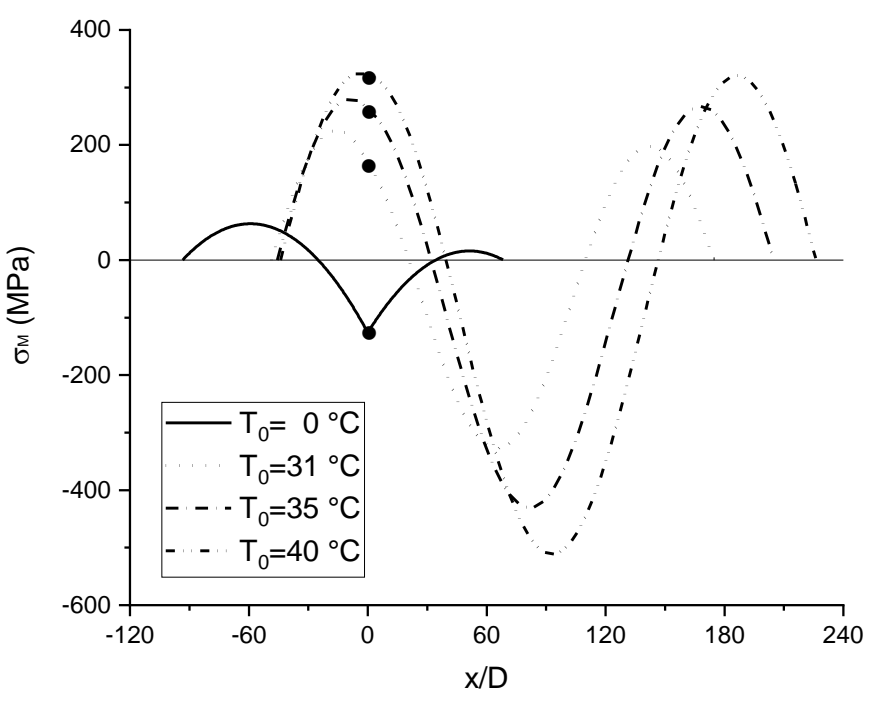

(b)

Fig. 5 Influence of temperature difference on configuration. (a) Deformed shapes. (b) Bending stress. $h=1.5 D$. The dots represent the touchdown point at the step.

The locations of maximum displacement, denoted by $x_{w m}$, and of the three extreme values of the axial compressive stress can be obtained by $\frac{d w}{d x}=0$ and $\frac{d^{3} w}{d x^{3}}=0$, respectively, and are shown in Fig. 6. These locations may be obtained on either deflections $w_{1}$ or $w_{2}$ depending on the step height and the temperature difference $T_{0}$. Once the locations are obtained, the extreme values can be calculated from the solutions for $w_{1}$ or $w_{2}$.

We see in Fig. 6 that the locations of maximum displacement and extreme values of the axial compressive stress change with increasing temperature difference and that the locations of $w_{m}$ and $\sigma_{m 2}$ are almost coincident. Since the values of $x_{w m}, x_{\sigma m 2}$ and $x_{\sigma m 3}$ are all larger than zero, the locations of $w_{m}, \sigma_{m 2}$ and $\sigma_{m 3}$ all lie on the deflection $w_{2}$. So the values of $w_{m}, \sigma_{m 2}$ and $\sigma_{m 3}$ can be calculated based on the deflection $w_{2}$. The location of $\sigma_{m 1}$ is more complex (at least for the present parameters), as illustrated in Fig. 7(a). The corresponding values of $\sigma_{m 1}$ are shown in Fig. 7(b). In Fig. 7(a), $x_{\sigma m 1}<0$, indicating that the location of $\sigma_{m 1}$ lies on deflection $w_{1}$, while $x_{\sigma m 1}>0$, meaning that the location of $\sigma_{m 1}$ lies on deflection $w_{2}$. So $\sigma_{m 1}$ is obtained from $w_{1}$ for $T_{0}<T_{0}(a)$ and from $w_{2}$ for $T_{0}>T_{0}(b)$. For $T_{0}(a)<T_{0}<T_{0}(b)$ a close pair of maxima appear, straddling $x=0$, which is now a local minimum. As Fig. 7(b) shows, the corresponding stresses are extremely close. The larger of the two values is taken as $\sigma_{m 1}$. Fig. 8 shows the three stress maxima obtained as described above. We note that $\sigma_{m 1}$ and $\sigma_{m 3}$ are very close, and much smaller than $\sigma_{m 2}$. We conclude therefore that the maximum of the axial compressive stress along the pipeline occurs at location $x_{\sigma m 2}$, which is very close to the location of 
maximum displacement.

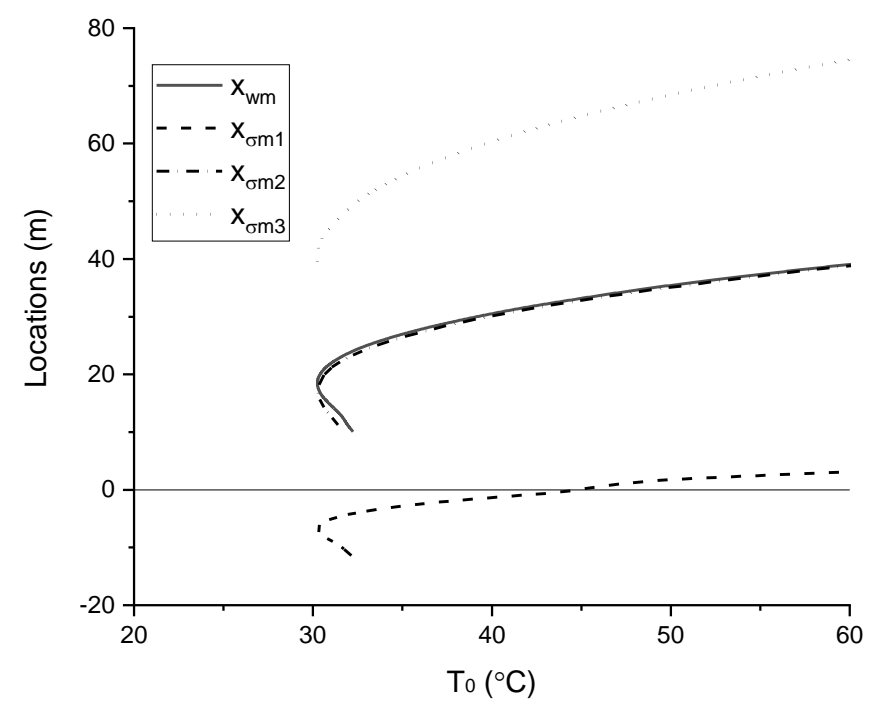

Fig. 6 The locations of maximum displacement and extrema of axial compressive stress. $h=1.5 D$.

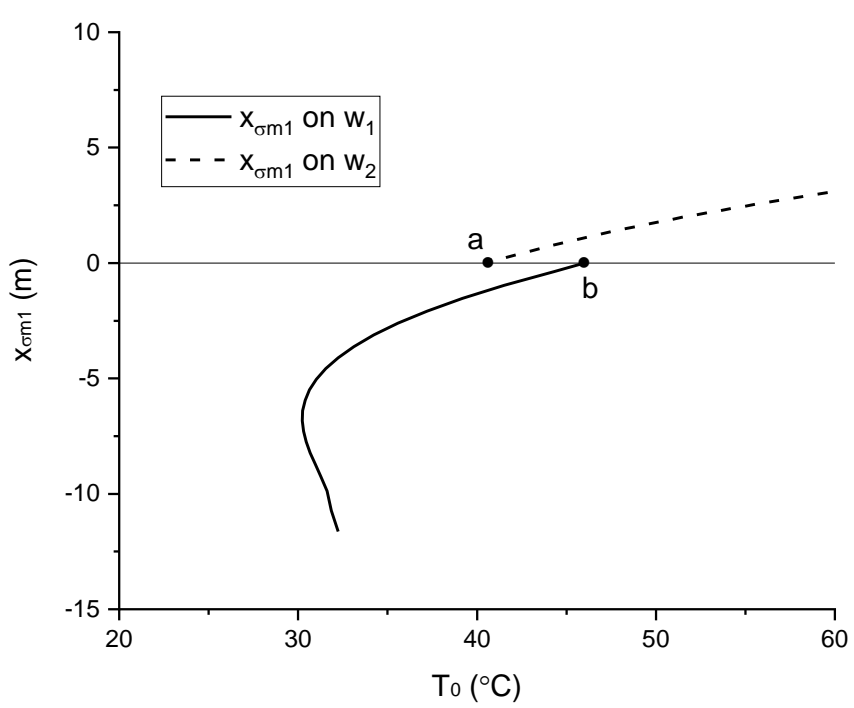

(a)

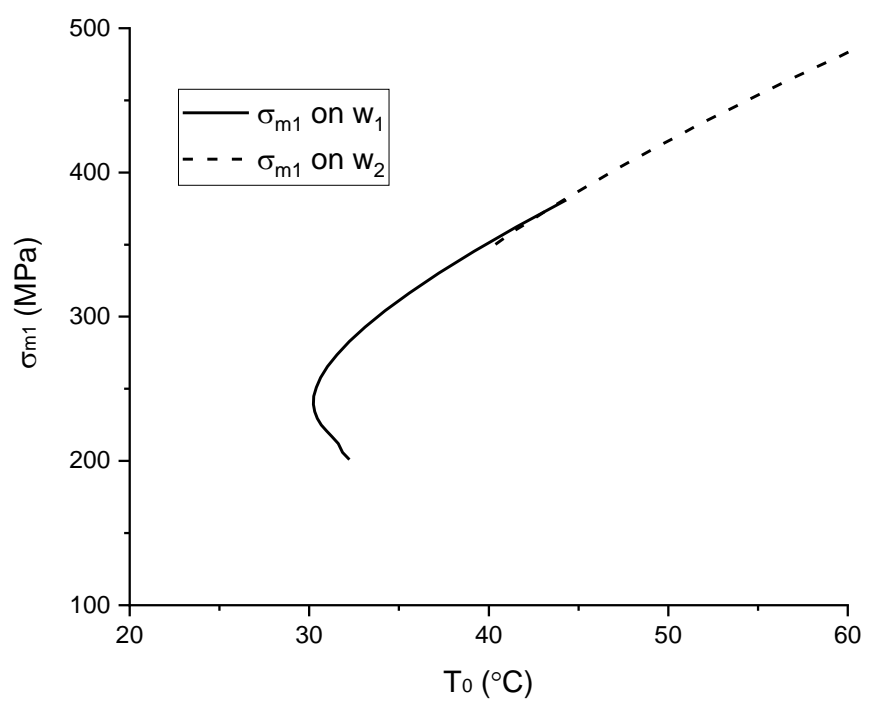

(b)

Fig. 7 (a) The locations of $\sigma_{m 1}$. (b) The corresponding values of $\sigma_{m 1} \cdot h=1.5 D$.

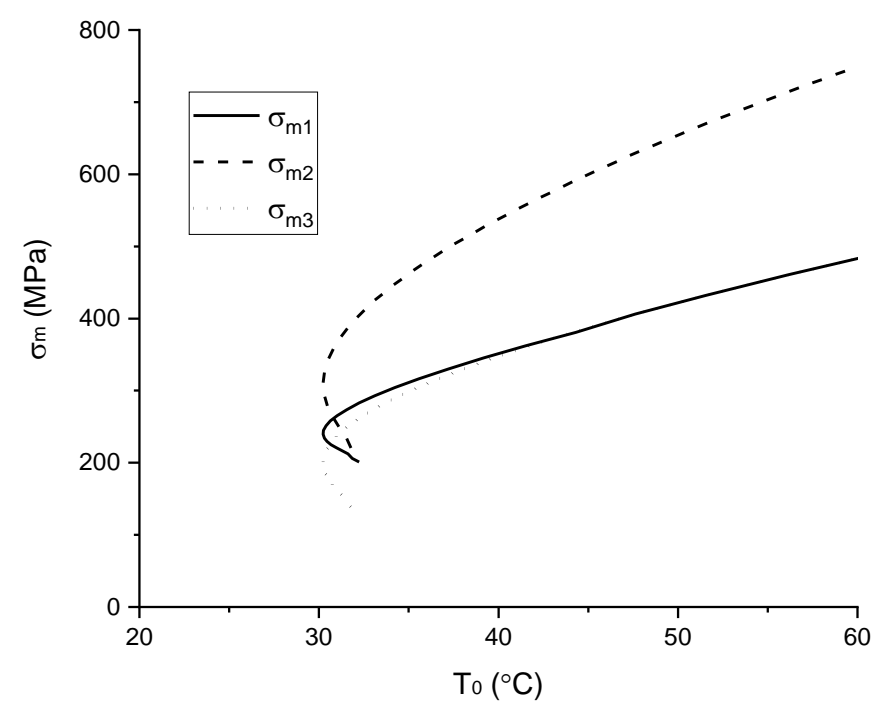

Fig. 8 The comparison of three extrema of axial compressive stress. $h=1.5 D$. 


\subsection{Analysis of typical post-buckling behaviour}

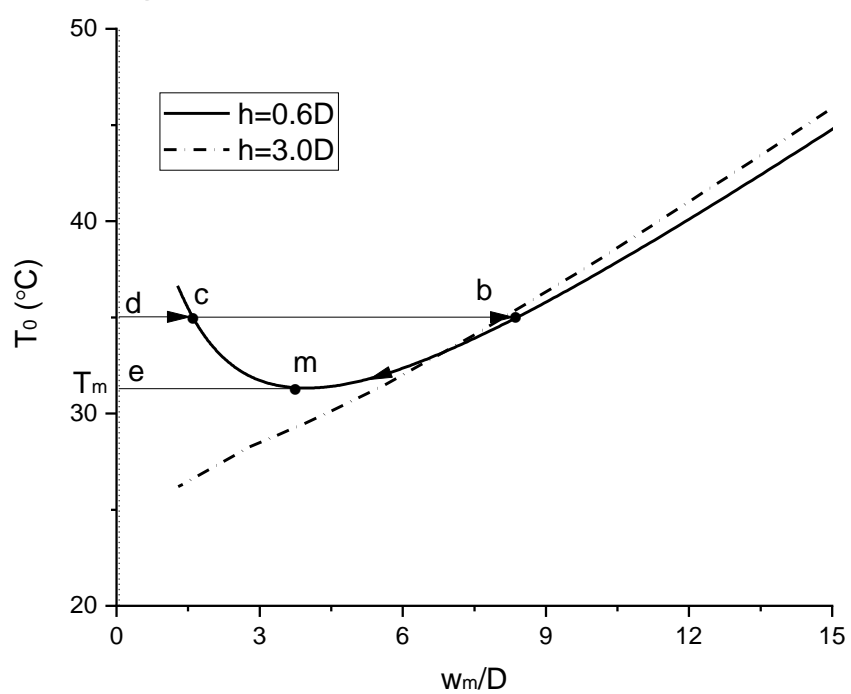

Fig. 9 Typical buckling paths. The dotted vertical line represents the maximum initial (pre-buckling) deflection.

In Fig. 9, two typical buckling paths show the typical post-buckling behaviour. $T_{m}$ is the minimum critical temperature difference. For the case of step height $h=0.6 D$, when the temperature difference is lower than $T_{m}$ only the trivial state exists and no upheaval buckling occurs. However, when the temperature difference is larger than $T_{m}$ two buckling states exist. One will be stable, the other will be unstable. We see in Fig. 9 that the maximum displacement $w_{m}$ increases with increasing temperature difference along branch $\mathrm{m}-\mathrm{b}$ and decreases with increasing temperature difference along branch $\mathrm{m}$-c. We therefore conclude that branch $m-b$ is stable and branch $m-c$ is unstable [19]. Taking $T_{0}=35^{\circ} \mathrm{C}$ as an example, when $T_{0}$ reaches $35^{\circ} \mathrm{C}$, the pipeline will remain unbuckled in the absence of a disturbance, corresponding to point $d$ in Fig. 9 . However, the deformed states $b$ and $c$ are available as well and a sufficiently large disturbance may cause a jump from state $d$ to the stable buckled state $b$, in a kind of snap-through buckling process. Conversely, under decreasing $T_{0}$ we expect a jump from the stable solution when it reaches state $m$ to state $e$ on the unbuckled branch (this time not requiring a disturbance). We will refer to this as snap-back buckling. However, we see in Fig. 9 that for large enough step height, such as $h=3.0 \mathrm{D}$, the minimum critical temperature difference $T_{m}$ disappears: $w_{m}$ decreases monotonically with decreasing temperature difference. We conclude that, for large enough step height, a stable buckled state exists for all temperatures and no snap-back buckling occurs. The snap-back buckling raises the possibility of hysteresis cycles $d-b-m-e-d \ldots$ under cyclic thermal loading.

\subsection{Parametric study}

Table 1. Design parameters.

\begin{tabular}{ccc}
\hline Parameter & Value & Unit \\
\hline External diameter $D$ & 323.9 & $\mathrm{~mm}$ \\
Wall thickness $t$ & 12.7 & $\mathrm{~mm}$ \\
Elastic modulus $E$ & 206 & $\mathrm{GPa}$ \\
Steel density $\rho$ & 7850 & $\mathrm{~kg} / \mathrm{m}^{3}$ \\
Coefficient of thermal expansion $\alpha$ & $1.1 \times 10^{-5}$ & $/{ }^{\circ} \mathrm{C}$ \\
Axial friction coefficient $\mu_{A}$ & 0.5 & --- \\
\hline
\end{tabular}

In this section, upheaval buckling of a typical pipeline resting on the seabed with step imperfection is analysed. The deformed shapes and bending stresses along the pipeline with different step height are analysed and discussed first. Then the influence of step height, axial soil resistance, wall thickness of the pipe on typical upheaval buckling behaviour is presented. We demonstrate the effect of step imperfection by employing the analytical formulation developed in Section 2 taking the parameters in Table 1 as a realistic case study. In this section, all the analysis is based on the stable branch. 


\subsubsection{The influence of step height}

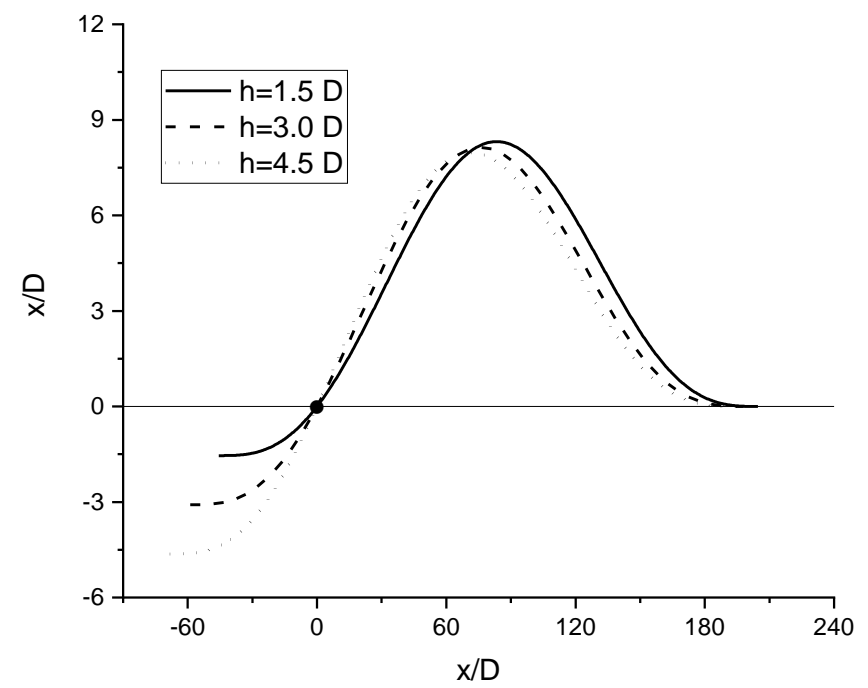

(a)

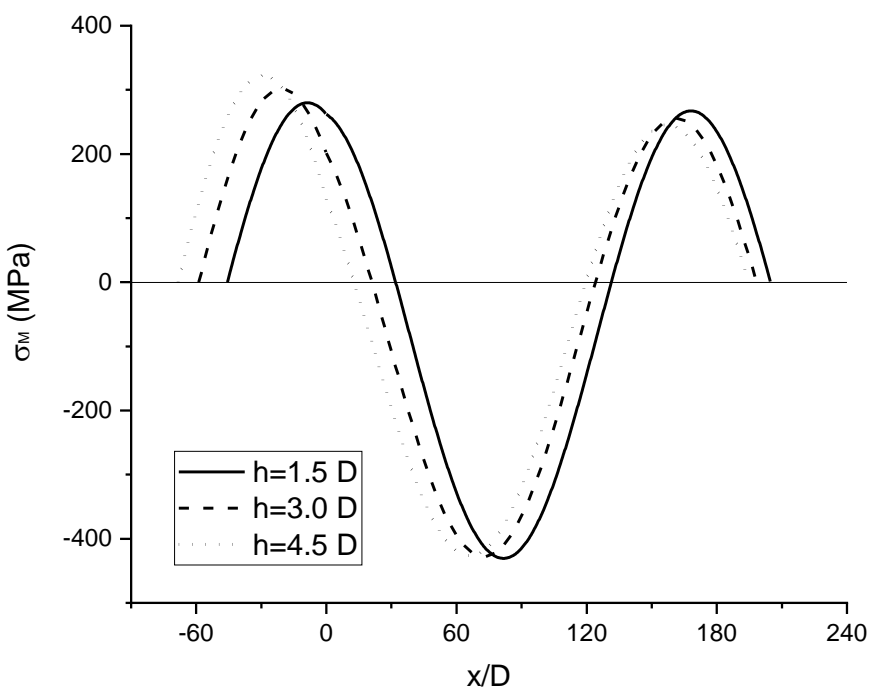

(b)

Fig. 10 Influence of step height on configuration. (a) Deformed shapes. (b) Bending stress. $T_{0}=35^{\circ} \mathrm{C}$. The dots represent the touchdown point at the step.

The deformed shapes and the corresponding bending stresses $\sigma_{M}$ along the buckled pipeline with different step height under the same operating temperature difference are presented in Fig. 10(a) and Fig. 10(b), respectively. It is seen that a smaller lobe is formed beneath the step while a larger lobe exists above the step for each buckled shape, as shown in Fig. 10(a). The smaller lobe enlarges while the larger lobe shrinks under an increase of step height. It is also seen in Fig. 10(a) that the locations of maximum displacement move close to the step under increasing step height while the maximum displacement decreases with increasing step height. In Fig. 10(b), we see that the locations of the extrema of bending stress change with increasing step height. Under increasing step height, the extremum of bending stress at $x=x_{\sigma m 1}$ increases, while the other two extrema of bending stress decrease.

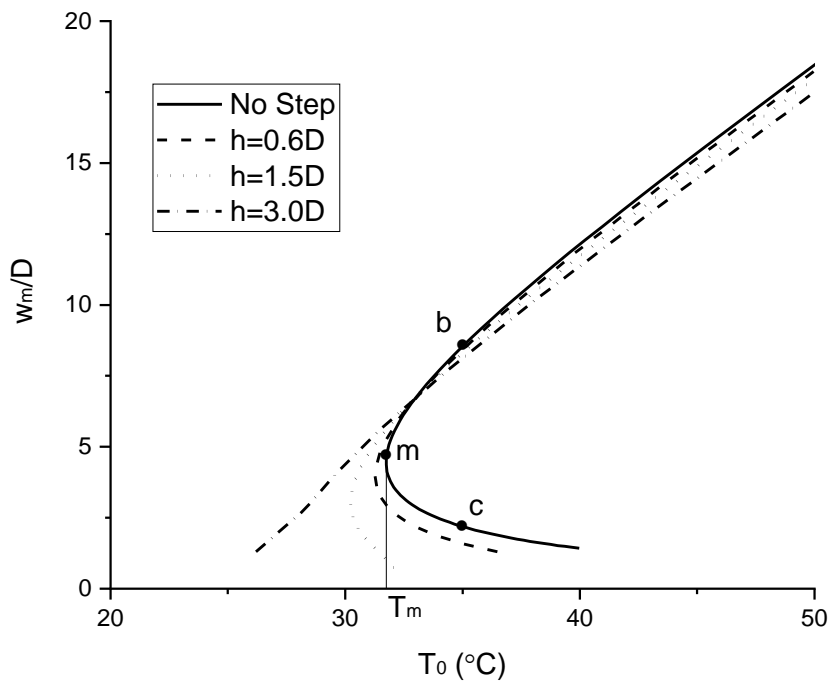

(a)

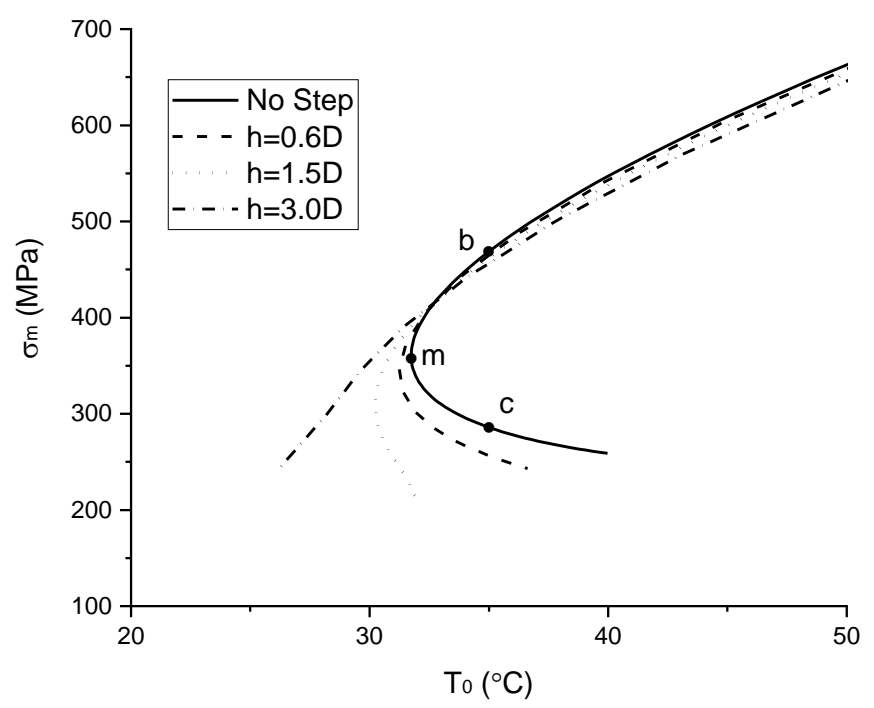

(b) 


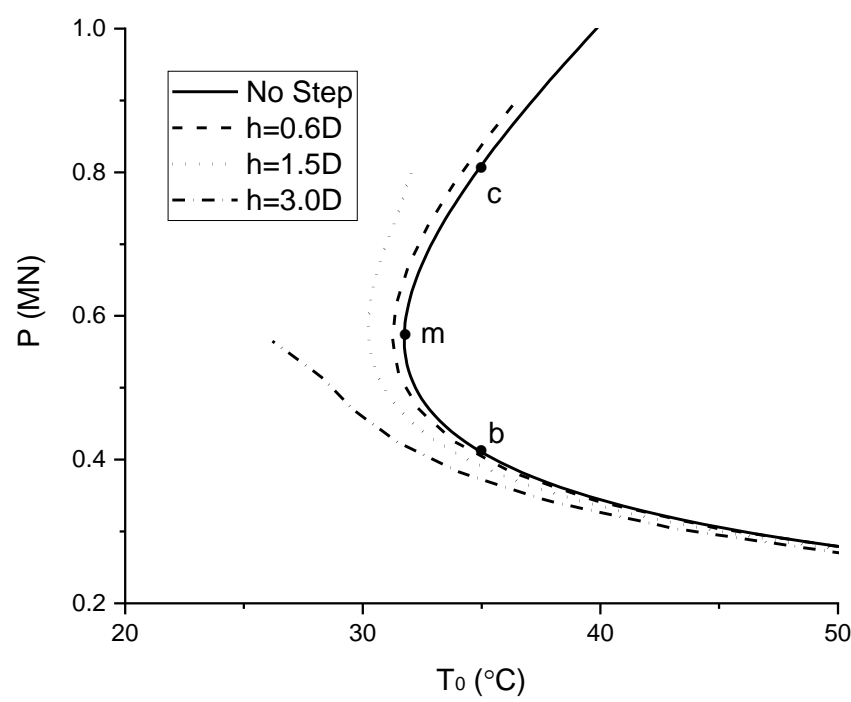

(c)

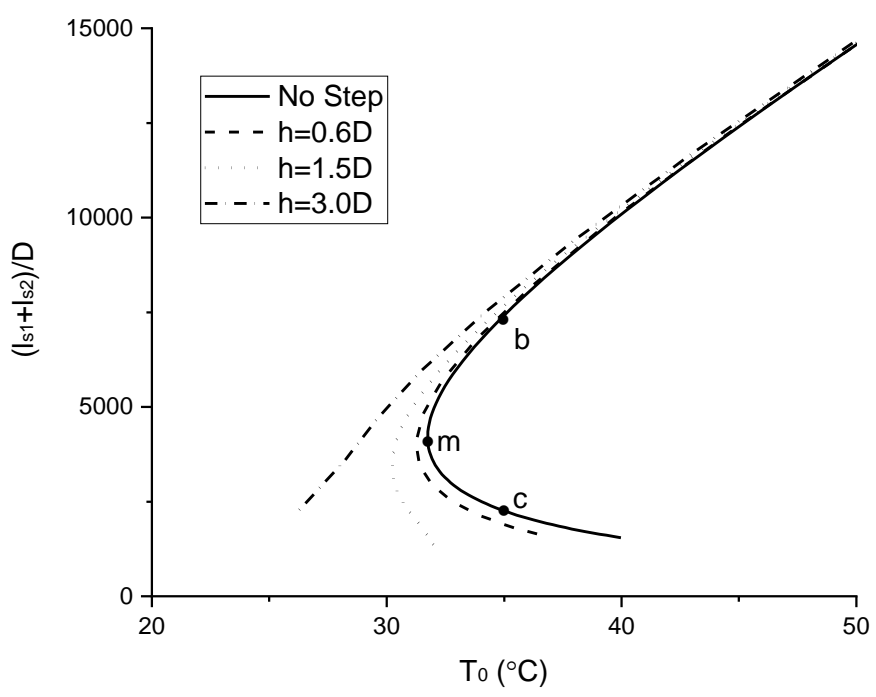

(e)

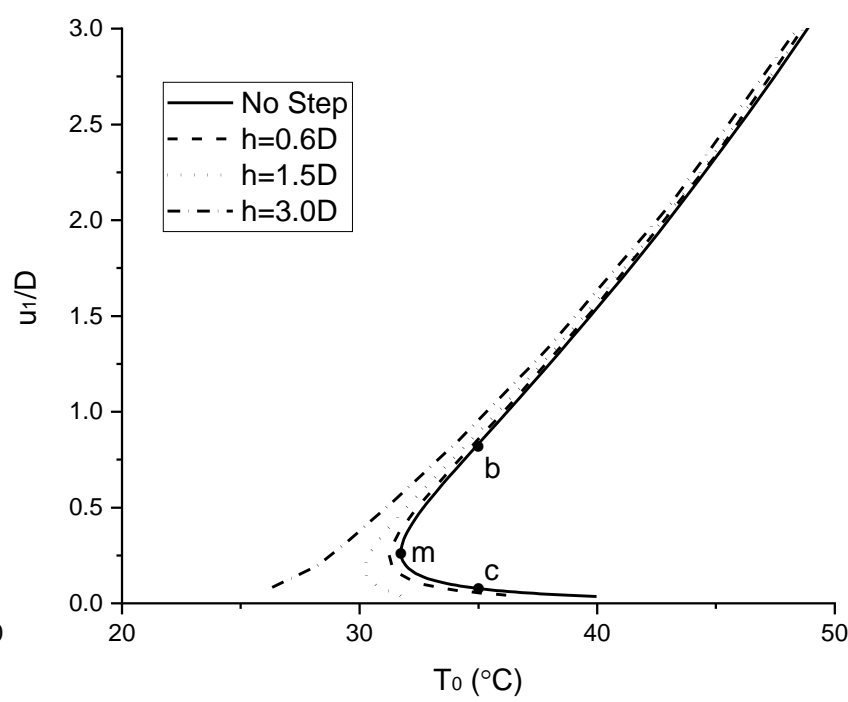

(d)

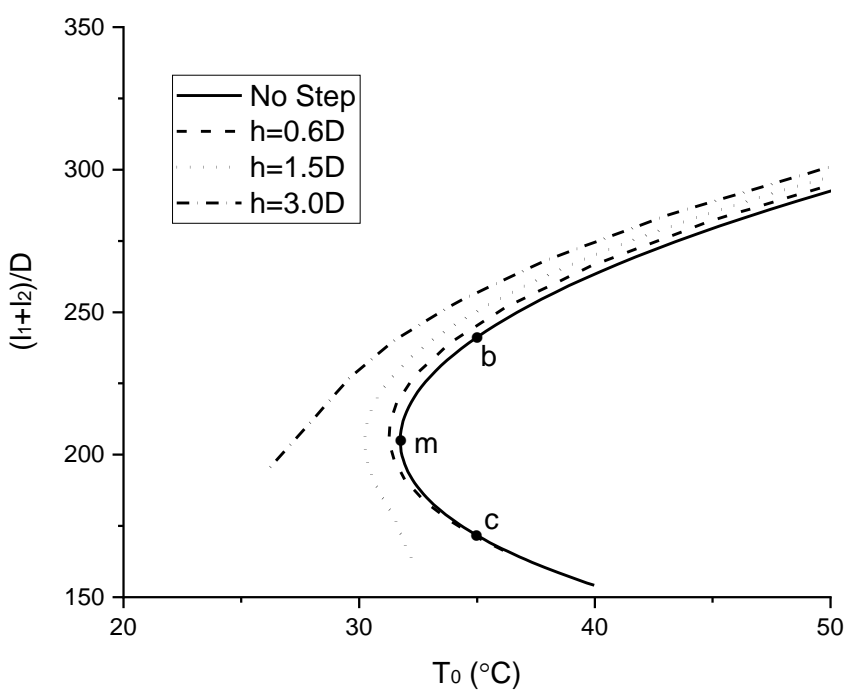

(f)

Fig. 11 Influence of step height on buckling behaviour. (a) Displacement amplitude $w_{m}$. (b) Maximum axial compressive stress $\sigma_{m}$. (c) Axial compressive force $P$. (d) Axial thermal expansion $u_{1}$. (e) Total feed-in length $\left(l_{s 1}+l_{s 2}\right)$. (f) Total buckled length $\left(l_{1}+l_{2}\right)$.

The influence of step height on typical buckling behaviour of upheaval buckling for pipelines with step imperfection is shown in Fig. 11. In Fig. 11(a), the minimum critical temperature difference $T_{m}$ decreases with increasing step height, so the pipeline is more likely to buckle vertically for larger step imperfection. For large enough step height, such as $h=3.0 D$, the minimum critical temperature difference $T_{m}$ disappears, which means that $w_{m}$ will increase gradually with increasing temperature difference and no snap-back buckling will occur. However, for smaller step height, such as $h=0.6 D$, upheaval buckling may happen when the temperature difference is larger than the minimum critical temperature difference $T_{m}$ and snap-back may happen at $T_{m}$. Under the same temperature difference, the displacement amplitude decreases with increasing step height in the stable buckled state. From Fig. 11(b), the maximum axial compressive stress $\sigma_{m}$ increases with increasing temperature difference. Under the same temperature difference, the maximum axial compressive stress $\sigma_{m}$ decreases with increasing step height. From Fig. 11(c), we see that the axial compressive force decreases with increasing temperature difference, while under the same temperature difference, the axial compressive force decreases with increasing step height.

From Fig. 11(d) and Fig. 11(e) we see that the axial thermal expansion $u_{1}$ and the total feed-in length $l_{s 1}+l_{s 2}$ increase with the increase of step height. However, both the buckling amplitude and maximum axial compressive stress decrease with 
increasing step height under the same temperature difference, as shown in Fig. 11(a) and Fig. 11(b). Two reasons lead to the decrease of maximum axial compressive stress with increasing step height. The first reason is that the axial compressive force and the corresponding stress induced by it reduces with increasing step height, as shown in Fig. 11(c) and Fig. 12(a). The second reason is that, with increasing step height, the deflection is more benign since $w_{m}$ reduces while the total buckled length $l_{1}+l_{2}$ increases (see Fig. 11(a) and Fig. 11(f)). So the maximum bending stress induced by the maximum bending moment reduces with increasing step height, as shown in Fig. 12(b).

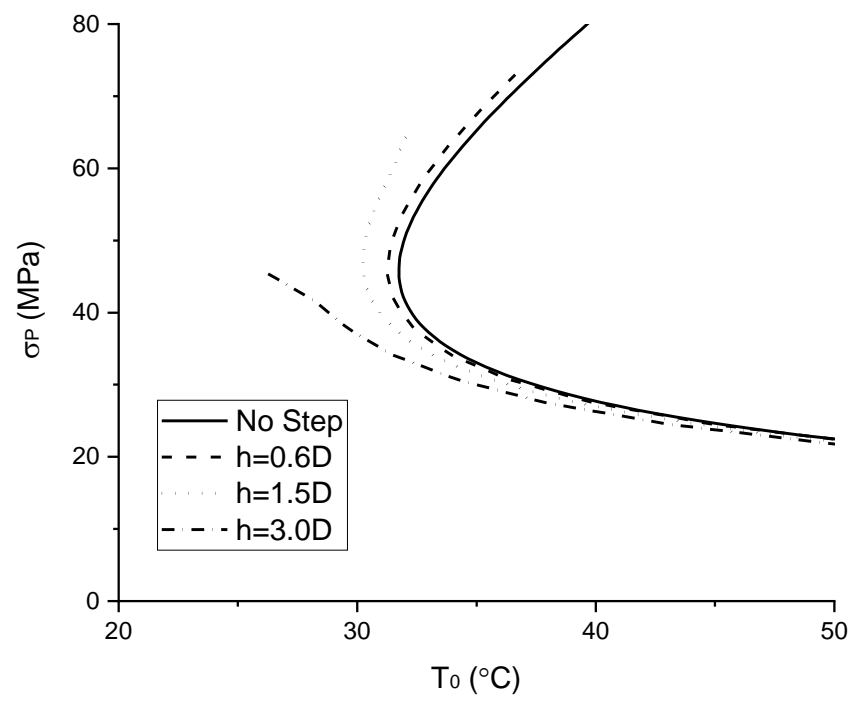

(a)

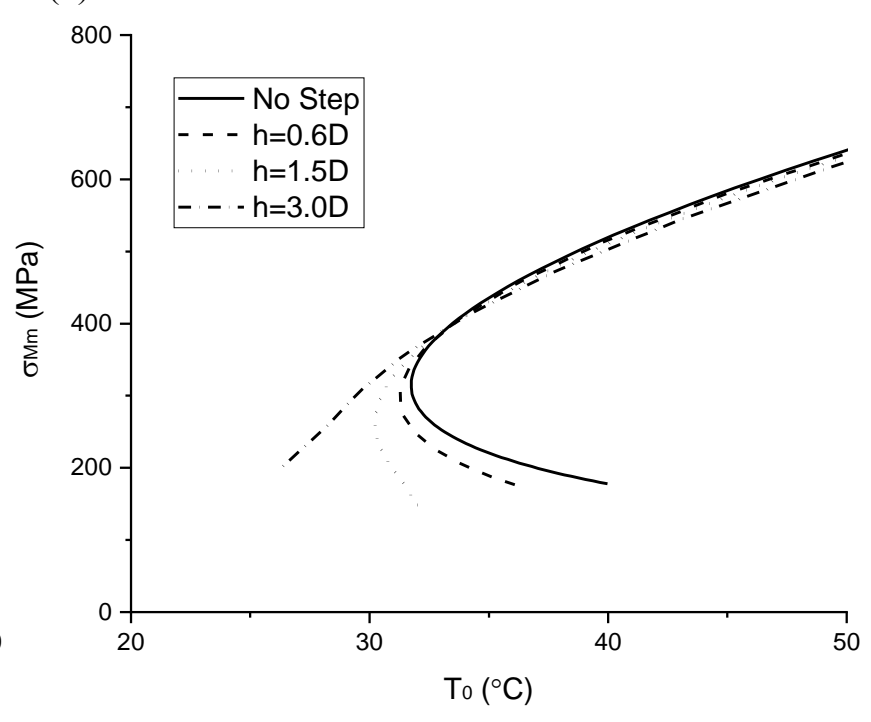

(b)

Fig. 12 The influence of step height on the component of maximum axial compressive stress. (a) Stress indeced by axial compressive force $P$. (b) Stress induced by maximum bending moment.

\subsubsection{The influence of axial soil resistance}

The influence of axial soil resistance on typical buckling behaviour of upheaval buckling for pipelines with step imperfection is shown in Fig. 13. The minimum critical temperature difference $T_{m}$ increases with increasing axial soil resistance (see Fig. 13(a)), since it is more difficult for the pipeline to obtain enough axial thermal expansion due to the larger axial soil resistance (see Fig. 13(d)). Both the buckling amplitude and total buckled length $l_{1}+l_{2}$ decrease with increasing axial soil resistance under the same temperature difference, as shown in Fig. 13(a) and Fig. 13(f), while the axial thermal expansion decreases with increasing axial soil resistance, as shown in Fig. 13(d). So we see that the buckled shape shrinks with increasing axial soil resistance under the same temperature difference. From Fig. 13(b), the maximum axial compressive stress decreases with increasing axial soil resistance, even though the axial compressive force $P$ and the stress induced by it increase with increasing axial soil resistance under the same temperature difference (see Fig. 13(c) and Fig. 14(a)). Despite the increase of $P$, the maximum axial compressive stress reduces because the maximum bending stress induced by the bending moment reduces with increasing axial soil resistance, as shown in Fig. 14(b). Furthermore, larger axial soil resistance will result in smaller axial thermal expansion and total feed-in length, as shown in Fig. 13(d) and Fig. 13(e), leading to a more benign deflection. 


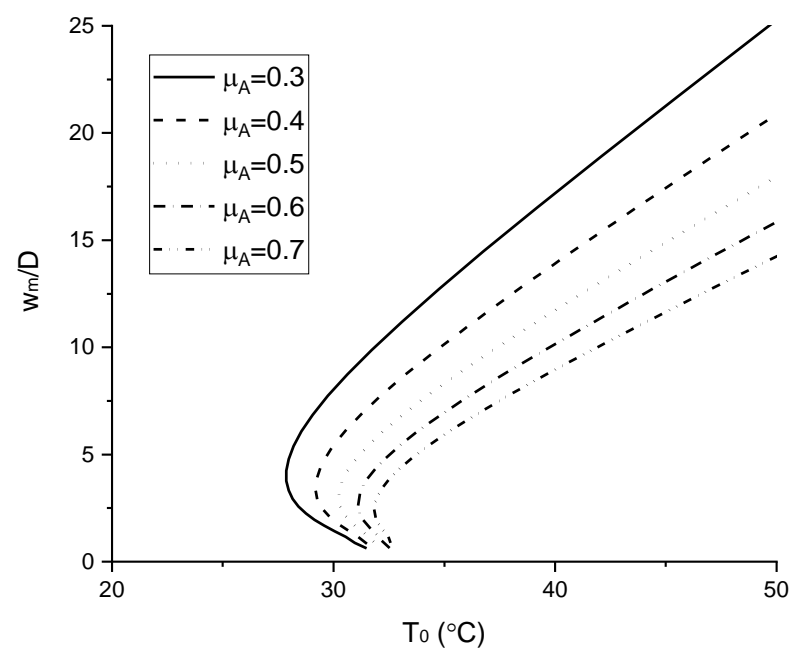

(a)

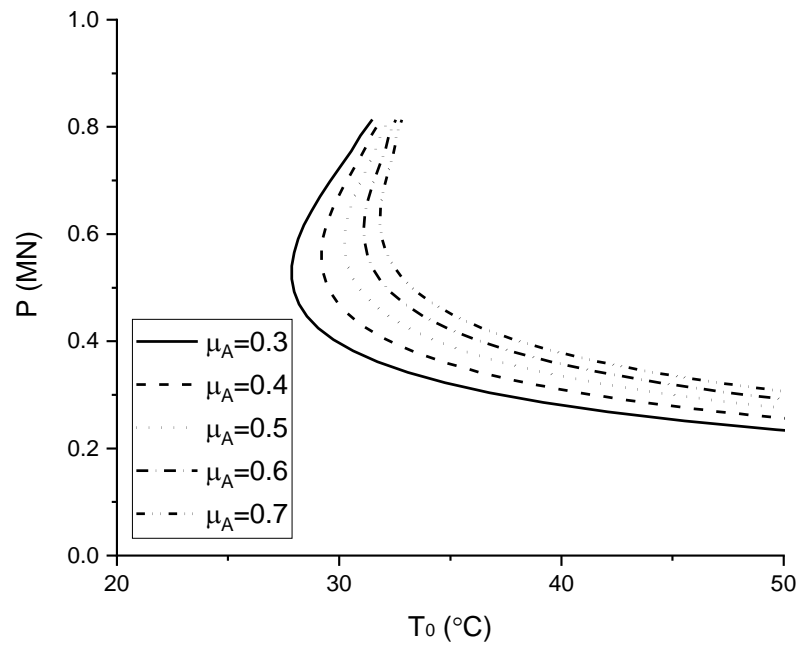

(c)

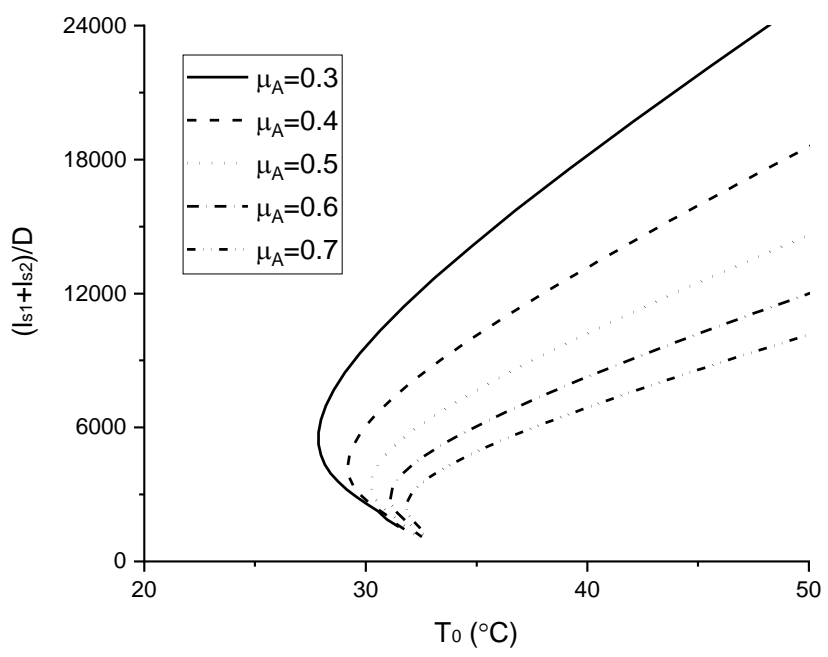

(e)

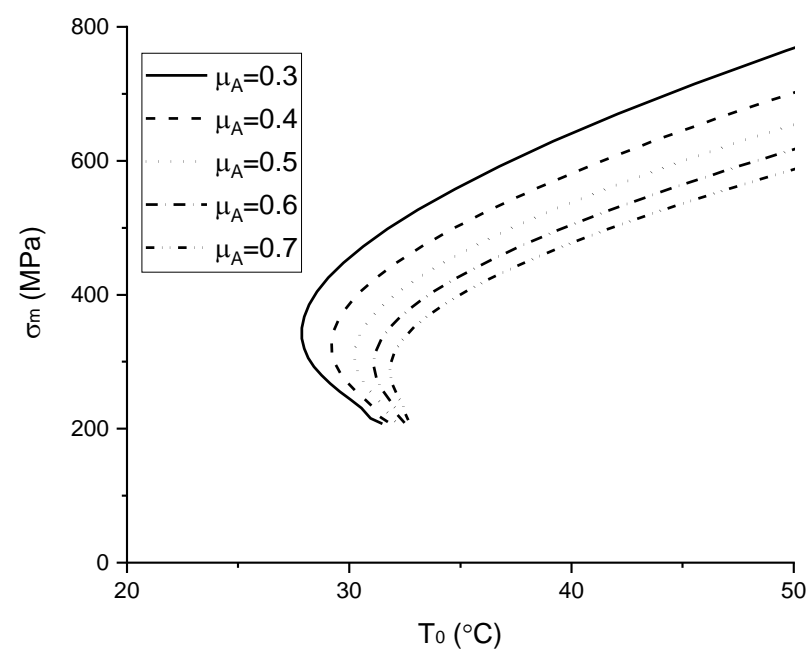

(b)

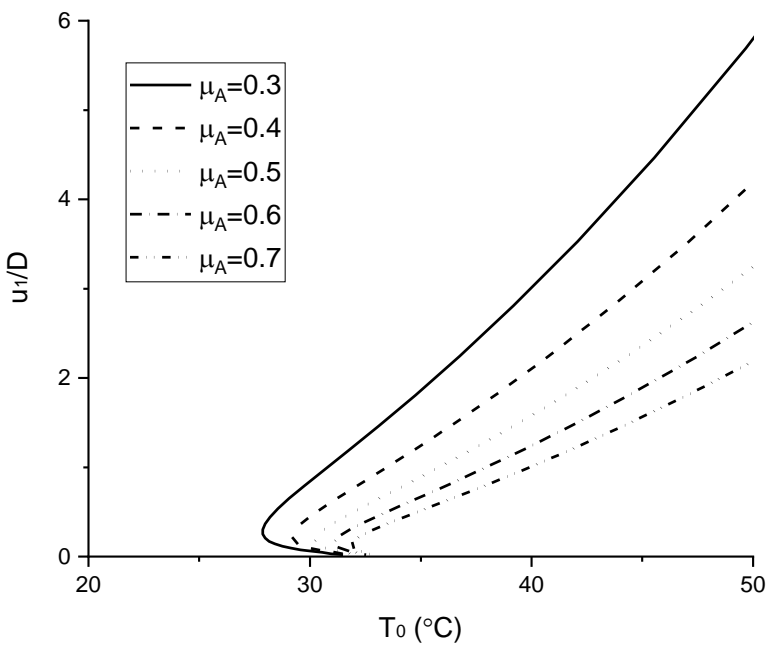

(d)

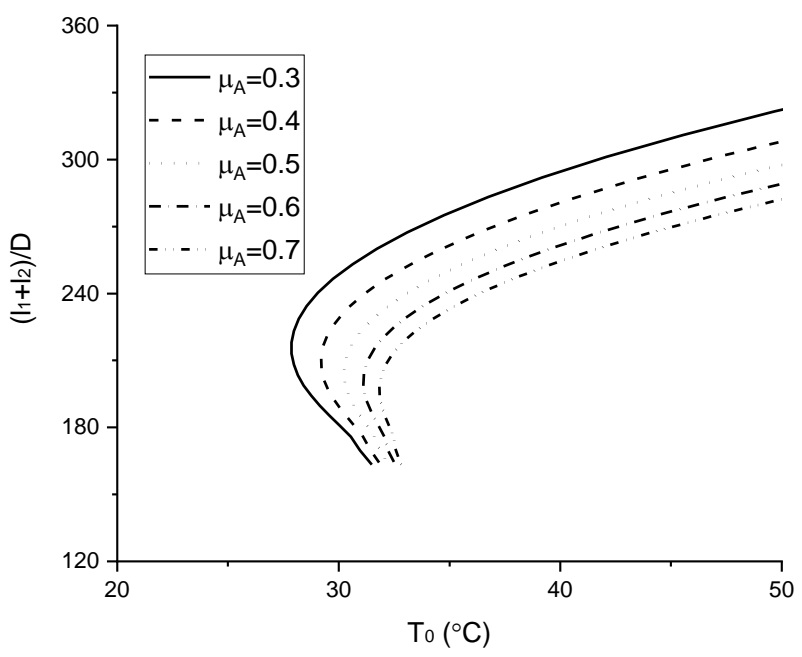

(f)

Fig. 13 Influence of axial soil resistance on buckling behaviour. (a) Displacement amplitude $w_{m}$. (b) Maximum axial compressive stress $\sigma_{m}$. (c) Axial compressive force P. (d) Axial thermal expansion $u_{1}$. (e) Total feed-in length $\left(l_{s 1}+l_{s 2}\right)$. (f) Total buckled length $\left(l_{1}+l_{2}\right) . h=1.5 D$. 


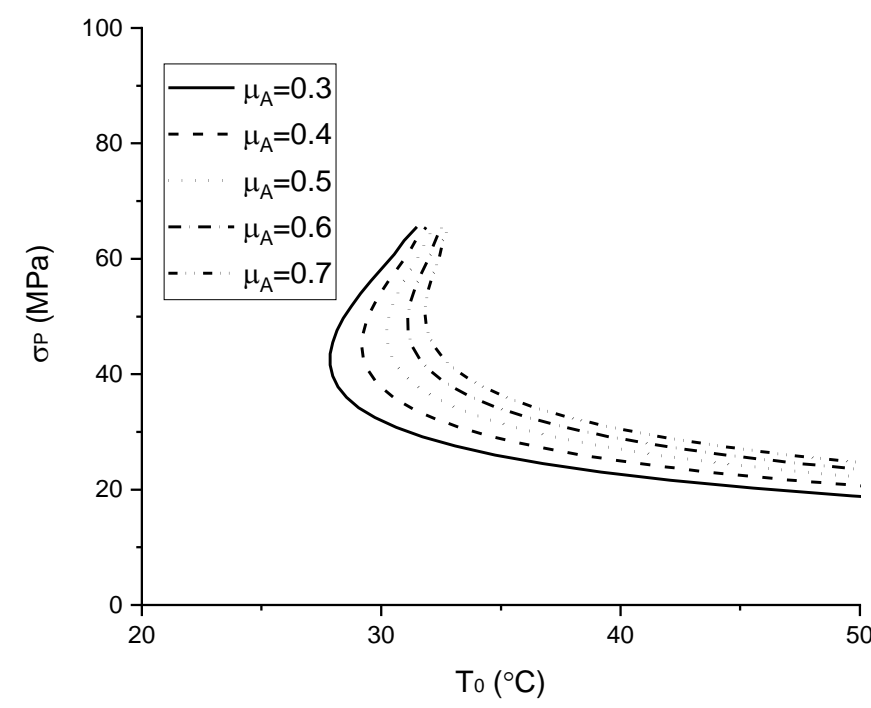

(a)

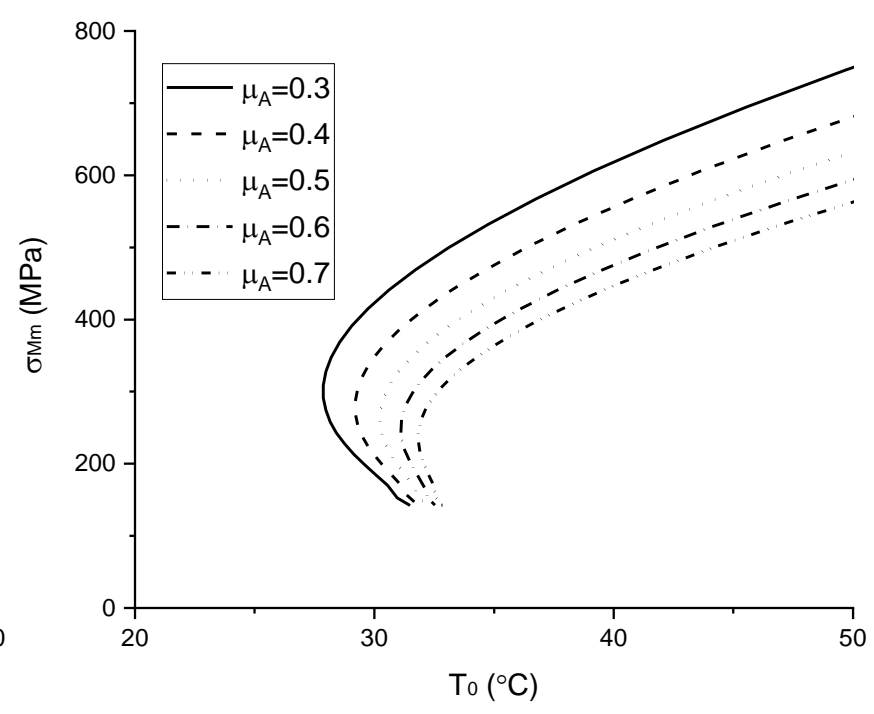

(b)

Fig. 14 The influence of axial soil resistance on the component of maximum axial compressive stress. (a) Stress indeced by axial compressive force $P$. (b) Stress induced by maximum bending moment. $h=1.5 \mathrm{D}$.

\subsubsection{The influence of wall thickness}

The influence of wall thickness on typical buckling behaviour of upheaval buckling for pipelines with step imperfection is shown in Fig. 15. In Fig. 15(a), the minimum critical temperature difference $T_{m}$ decreases with increasing wall thickness. The wall thickness doesn't have much influence on the buckling displacement amplitude. For larger wall thickness, the buckling amplitude is larger at lower temperature difference while it becomes smaller at higher temperature difference due to its smaller rate of increase. The total buckled length $l_{1}+l_{2}$ decreases (see Fig. 15(f)); however, the axial thermal expansion $u_{1}$ and total feed-in length $l_{s 1}+l_{s 2}$ increase with increasing wall thickness (see Fig. 15(d) and Fig. 15(e)). From Fig. 15(c), the axial compressive force increases with increasing wall thickness; however, the stress induced by axial compressive force $P$ decreases with increasing wall thickness due to the increase of the area of the cross-section (see Fig. 16(a)). And the maximum axial compressive stress increases (see Fig. 15(b)) with increasing wall thickness because of the increase of maximum bending stress induced by the maximum bending moment (see Fig. 16(b)). So we conclude that pipelines with larger wall thickness are more prone to upheaval buckling. The maximum axial compressive stress during the post-buckling stage becomes larger for pipelines with larger wall thickness. It is therefore advisable to reduce the wall thickness of the pipeline when a step imperfection is present.

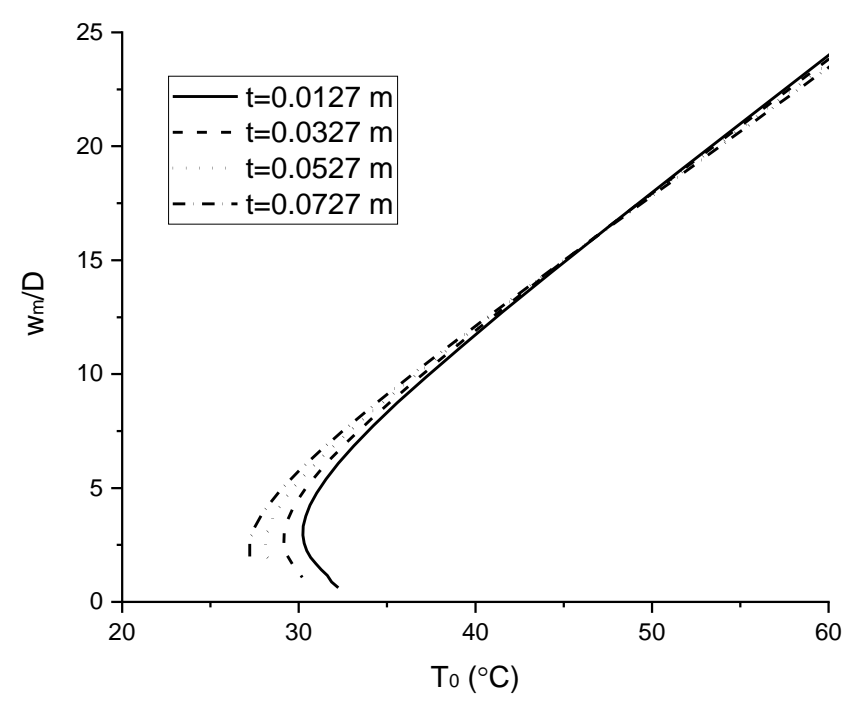

(a)

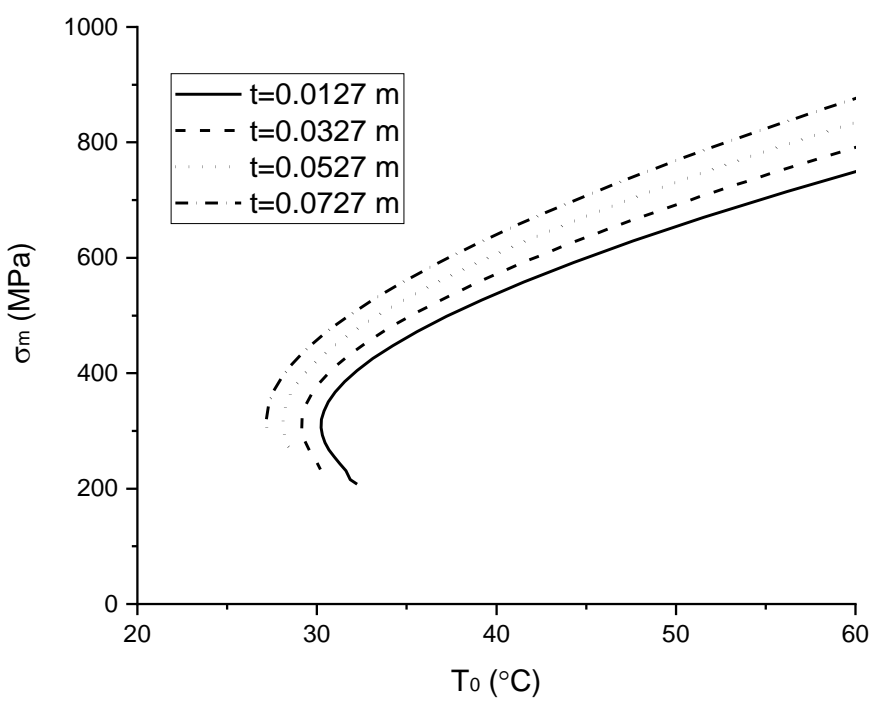

(b) 


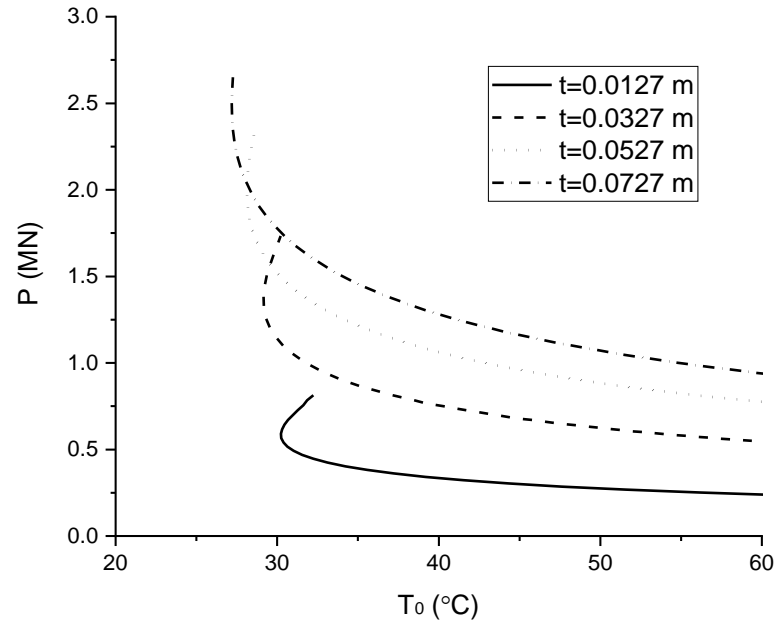

(c)

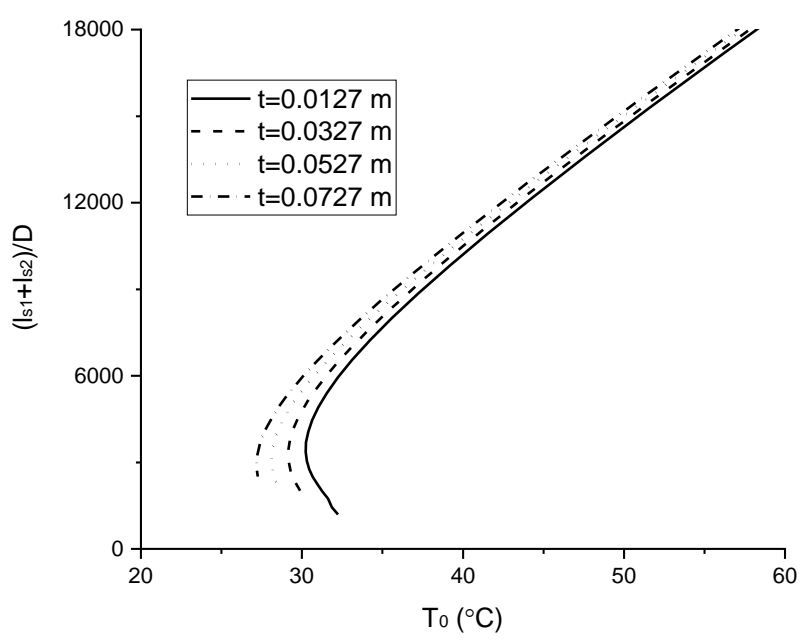

(e)

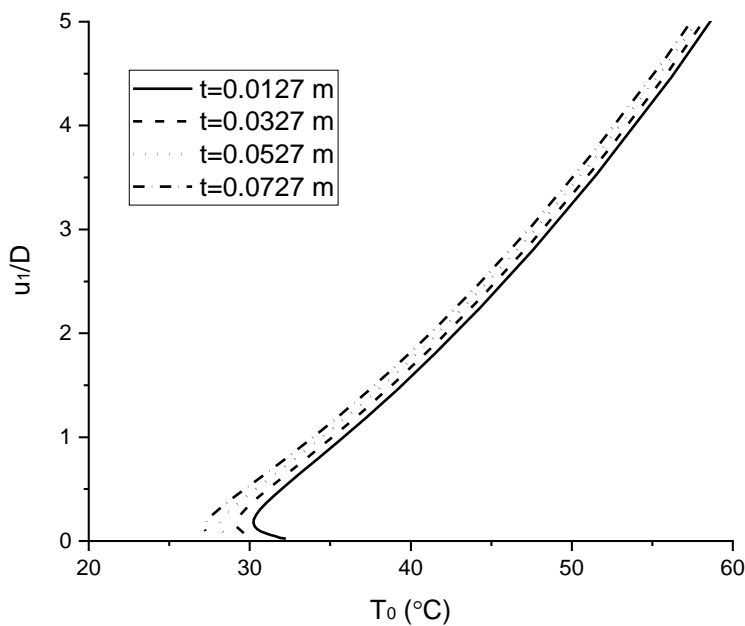

(d)

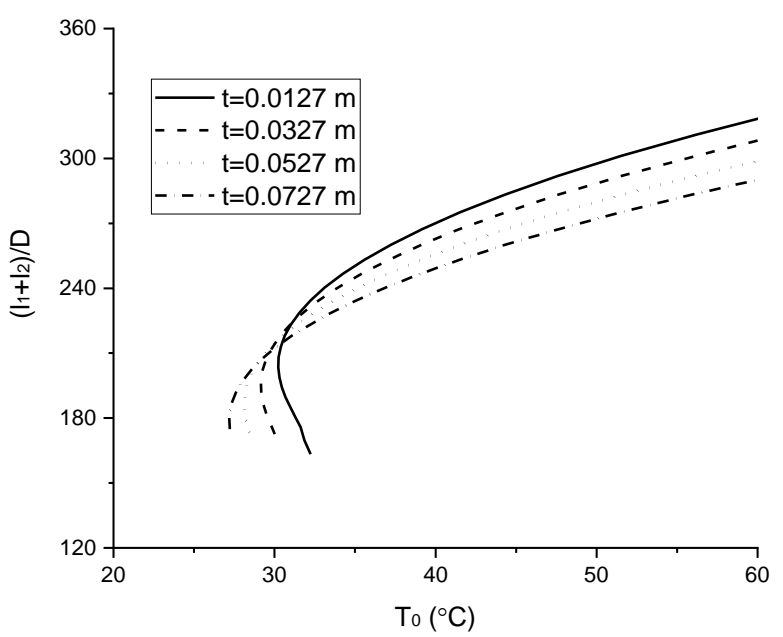

(f)

Fig. 15 Influence of wall thickness on buckling behaviour. (a) Displacement amplitude $w_{m}$. (b) Maximum axial compressive stress $\sigma_{m}$. (c) Axial compressive force P. (d) Axial thermal expansion $u_{1}$. (e) Total feed-in length $\left(l_{s 1}+l_{s 2}\right)$. (f) Total buckled length $\left(l_{1}+l_{2}\right) \cdot h=1.5 \mathrm{D}$.

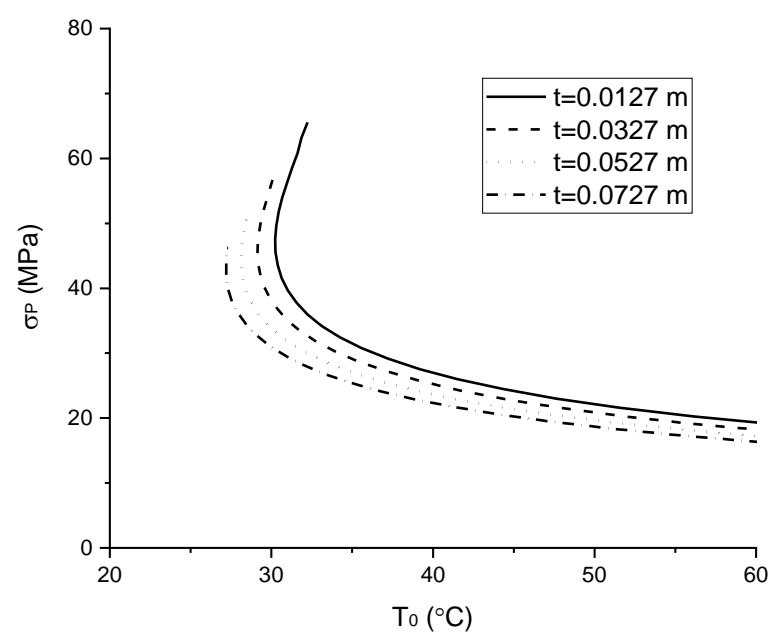

(a)

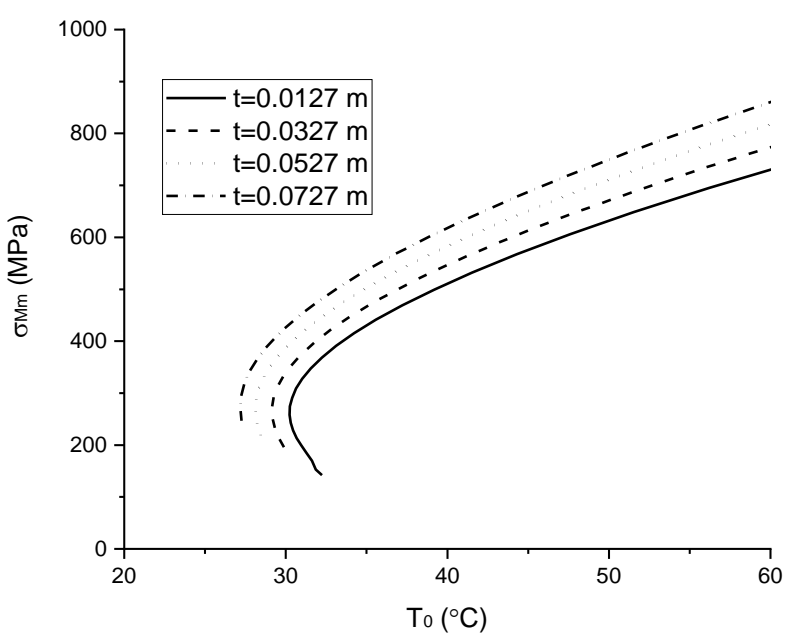

(b)

Fig. 16 The influence of wall thickness on the component of maximum axial compressive stress. (a) Stress indeced by axial compressive force $P$. (b) Stress induced by maximum bending moment. $h=1.5 D$. 


\subsubsection{The minimum critical temperature difference}

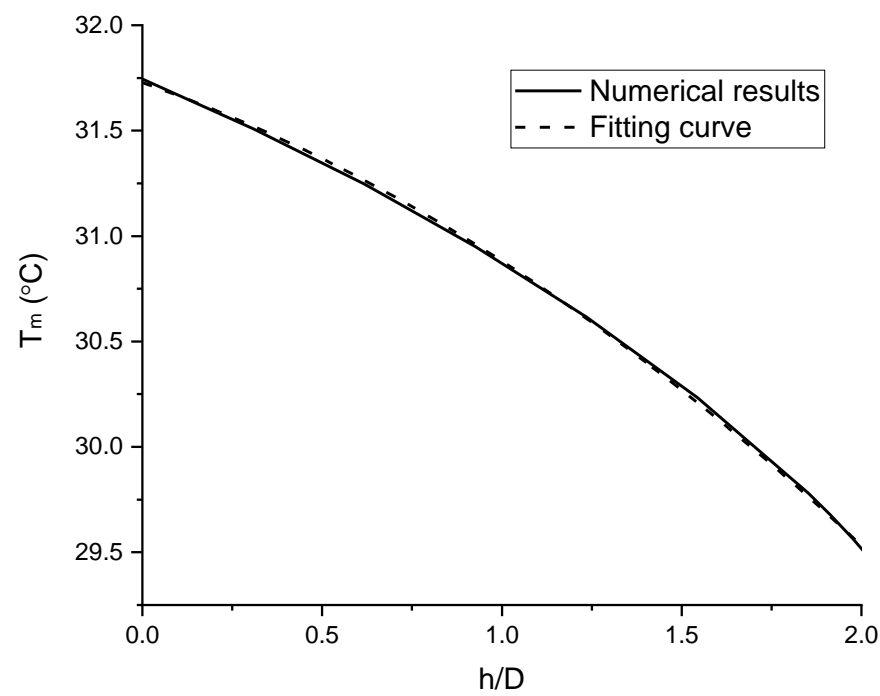

Fig. 17 The influence of step height on the minimum critical temperature difference.

The influence of step height on the minimum critical temperature difference $T_{\mathrm{m}}$ is presented in Fig. 17, in which a fitting curve is also included. The figure shows that the minimum critical temperature difference decreases with increasing step height. Its rate of decrease increases with increasing step height. The numerical results in the figure are fitted against the quadratic polynomial $y=d_{1} x^{2}+d_{2} x+d_{3}$, with coefficients $d_{1}=-0.25105, d_{2}=-0.59557$ and $d_{3}=31.72735$. The fitting parameters $d_{i}(i=1-3)$ of course depend on the parameters of the pipeline; further parametric studies should be carried out to obtain universal fitting parameters.

\subsection{Comparison between step and symmetric prop imperfection}

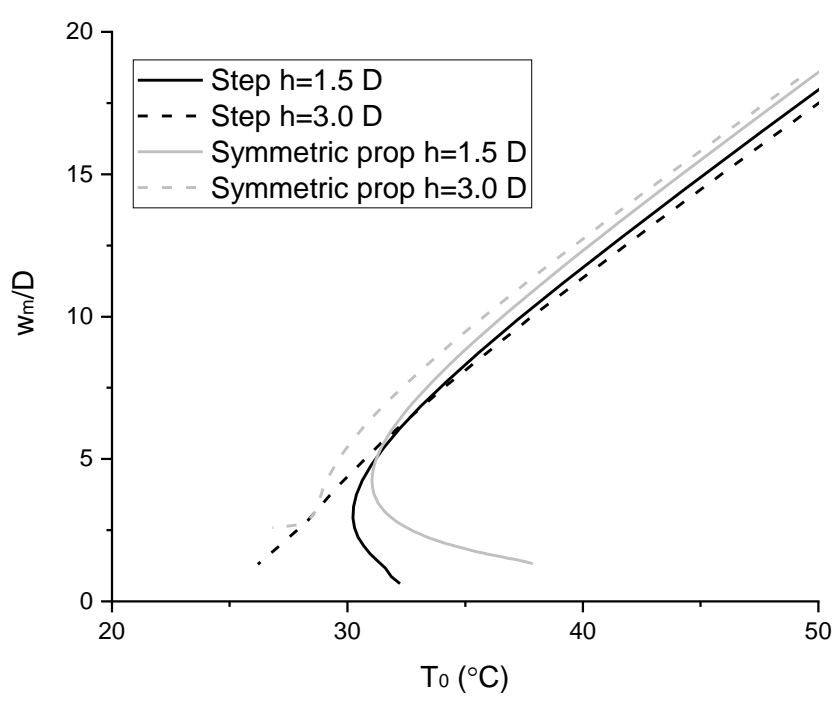

(a)

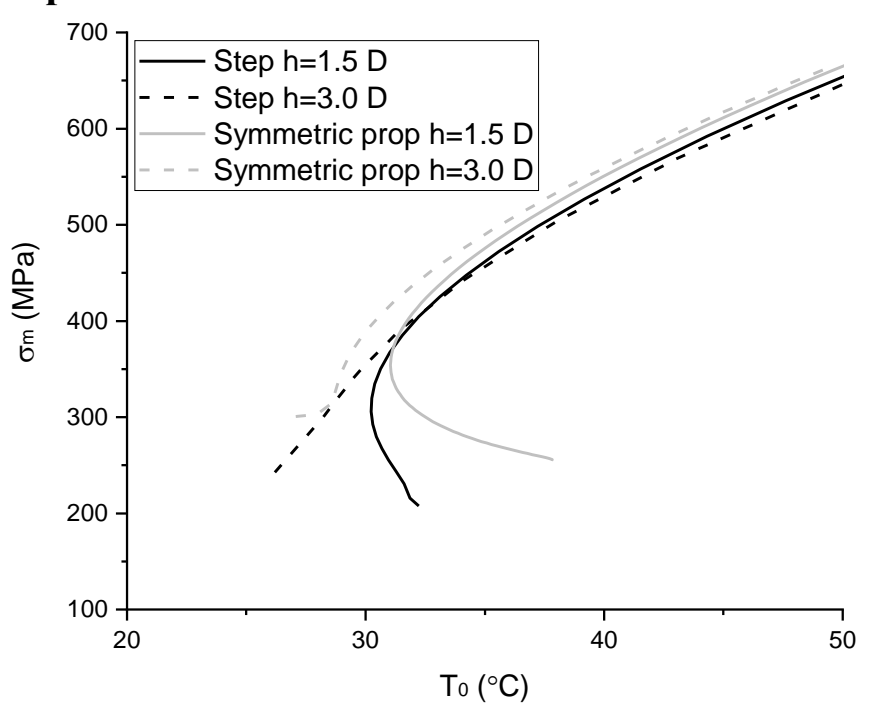

(b)

Fig. 18 Comparison between step and symmetric prop imperfection. (a) Maximum displacement $w_{m}$. (b) Maximum axial

$$
\text { compressive stress } \sigma_{m} \text {. }
$$

Fig. 18 compares results for a step imperfection with results for a symmetric prop imperfection. Both the maximum displacement and the maximum axial compressive stress are smaller for a pipeline with step imperfection than for a pipeline with symmetric prop imperfection, at the same imperfection amplitude and under the same temperature difference. For a pipeline with symmetric prop imperfection, both the maximum displacement and the maximum axial compressive stress increase with increasing imperfection amplitude, while for a pipeline with step imperfection both decrease with increasing imperfection amplitude. We can therefore conclude that a pipeline with step imperfection is safer than a pipeline with 
symmetric prop imperfection for the same imperfection amplitude. The imperfection amplitude should be reduced to the utmost to minimise the maximum axial compressive stress in case of a symmetric prop imperfection.

\section{Conclusions}

We have derived analytical solutions of upheaval buckling for subsea pipelines with topographic step imperfection. First, the accurate locations of maximum displacement and three extrema of axial compressive stress were obtained. The three stress extrema were compared to determine which of them is the maximum axial compressive stress. Then the typical post-buckling behaviour was analysed and the branch of stable solutions identified. Finally, a detailed parametric analysis was carried out. From our parametric analysis the following conclusions can be drawn:

(i) The analysis of typical post-buckling behaviour reveals that snap-through-type upheaval buckling may occur for all step heights but that snap-back buckling from the buckled state back to the unbuckled state under decreasing temperature only occurs for sufficiently small step heights. For such step heights there exists therefore the possibility of repeated jumps into and out of upheaval buckles if the pipeline experiences cyclic thermal loading conditions. In practice, subsea pipelines are subject to regular start-ups and shut-downs. For relatively small steps these may thus lead to hysteretic upheaval snap buckling.

(ii) Upheaval buckling occurs more easily for pipelines with step imperfection. The minimum critical temperature difference decreases further with increasing step height. Both the maximum displacement and the maximum axial compressive stress are smaller than those for pipelines without step imperfection and decrease further with increasing step height under the same temperature difference.

(iii) With increasing axial soil resistance, the minimum critical temperature difference increases. However, both the maximum displacement and the maximum axial compressive stress decrease. So a possible method to control buckling behaviour of pipelines with step imperfection is to increase the axial soil resistance.

(iv) Pipelines with step imperfections are more prone to upheaval buckling for larger wall thickness, which is contrary to the case without step imperfection. Also, the maximum axial compressive stress increases with increasing wall thickness. So the pipeline is more dangerous with larger wall thickness when a step imperfection exists.

(v) Pipelines with step imperfection are safer than those with symmetric prop imperfection for the same imperfection amplitude.

\section{Acknowledgments}

The authors would like to acknowledge that the work described in this paper was funded by the International Postdoctoral Fellowship Program from the China Postdoctoral Council (No. 20180049).

\section{Appendix A.}

$$
\begin{aligned}
& A_{1}=\frac{h}{l_{1}^{3}}-\frac{l_{1} q}{8 E I} \\
& A_{2}=\frac{3 h}{l_{1}{ }^{2}}-\frac{l_{1}^{2} q}{8 E I} \\
& A_{3}=\frac{3 h}{l_{1}}-\frac{l_{1}^{3} q}{24 E I} \\
& A_{4}=0 \\
& A_{5}=\frac{q\left(l_{1}^{2}+2 l_{2}^{2}\right)}{24 E I l_{2}}-\frac{h}{l_{2} l_{1}^{2}} \\
& A_{6}=\frac{3 h}{l_{1}^{2}}-\frac{l_{1}^{2} q}{8 E I} \\
& A_{7}=\frac{3 h}{l_{1}}-\frac{l_{1}^{3} q}{24 E I} \\
& A_{8}=0
\end{aligned}
$$




$$
\begin{aligned}
B_{1} & =\frac{\sin y_{1}\left(2 E I h \lambda^{4}+q y_{1}^{2}-2 q\right)+2 q y_{1} \cos y_{1}}{2 E I \lambda^{4}\left(\sin y_{1}-y_{1}\right)} \\
B_{2} & =\frac{2 \cot y_{1}\left(q-E I h \lambda^{4}\right)+q y_{1}\left(2-y_{1} \cot y_{1}\right)-2 q \csc y_{1}}{2 E I \lambda^{4}\left(y_{1} \csc y_{1}-1\right)} \\
B_{3} & =\frac{-2 E I h \lambda^{4}+q y_{1}^{2}-2 q y_{1} \sin y_{1}-2 q \cos y_{1}+2 q}{2 E I \lambda^{3} \sin y_{1}-2 E I \lambda^{3} y_{1}} \\
B_{4} & =-\frac{\sin y_{1}\left(2 E I h \lambda^{4}+q y_{1}^{2}-2 q\right)+2 q y_{1} \cos y_{1}}{2 E I \lambda^{4}\left(\sin y_{1}-y_{1}\right)} \\
B_{5} & =\frac{\sin y_{1}\left(2 E I h \lambda^{4}+q y_{1}^{2}-2 q\right)+2 q y_{1} \cos y_{1}}{2 E I \lambda^{4}\left(\sin y_{1}-y_{1}\right)} \\
B_{6} & =-\frac{\sin y_{1} \csc y_{2}\left(\cos y_{2}\left(2 E I h \lambda^{4}+q y_{1}^{2}-2 q\right)+2 q\right)-2 q y_{1} \csc y_{2}+2 q y_{1} \cos y_{1} \cot y_{2}}{2 E I \lambda^{4}\left(\sin y_{1}-y_{1}\right)} \\
B_{7}=\frac{2\left(\cot y_{1}\left(2 q-E I h \lambda^{4}\right)+\cot y_{2}\left(q-E I h \lambda^{4}\right)+E I h \lambda^{4} \csc y_{1}-2 q \csc y_{1}-q \csc y_{2}\right)+q y_{1}\left(-2 \cot y_{1} \cot y_{2}+2 \csc y_{1} \csc y_{2}-y_{1}\left(\cot y_{1}+\cot y_{2}+\csc y_{1}\right)+4\right)}{2 E I \lambda^{3}\left(y_{1} \csc y_{1}-1\right)} & \\
B_{8}= & -\frac{\sin y_{1}\left(2 E I h \lambda^{4}+q y_{1}^{2}-2 q\right)+2 q y_{1} \cos y_{1}}{2 E I \lambda^{4}\left(\sin y_{1}-y_{1}\right)}
\end{aligned}
$$

where, $y_{1}=\lambda l_{1}$ and $y_{2}=\lambda l_{2}$

\section{References}

[1] Bruton D, White DJ, Cheuk CY, Bolton MD, Carr M. Pipe-soil interaction behavior during lateral buckling, including largeamplitude cyclic displacement tests by the SAFEBUCK JIP (OTC-17944-MS). Offshore Technology Conference Houston, Texas 2006. p. 1-10.

[2] Karampour H. Effect of proximity of imperfections on buckle interaction in deep subsea pipelines. Marine Structures. 2018;59:444-57.

[3] Wang Z, Tang Y, Feng H, Zhao Z, Liu H. Model test for lateral soil resistance of partially embedded subsea pipelines on sand during large-amplitude lateral movement. J Coast Res. 2017;33:607-18.

[4] DNV-RP-F110. Global buckling of submarine pipelines structural design due to high temperature/high pressure. Det Norske Veritas; 2018.

[5] Hobbs RE. In-service buckling of heated pipelines. Journal of Transportation Engineering. 1984;110:175-89.

[6] Hobbs RE. Pipeline buckling caused by axial loads. Journal of Constructional Steel Research. 1981;1:2-10.

[7] Kerr AD. Analysis of Thermal Track Buckling in the Lateral Plane. 1978.

[8] Taylor N, Gan AB. Refined modelling for the vertical buckling of submarine pipelines. Journal of Constructional Steel Research. 1987;7:55-74.

[9] Taylor N, Gan AB. Refined modelling for the lateral buckling of submarine pipelines. Journal of Constructional Steel Research. 1986;6:143-62.

[10] Maltby TC, Calladine CR. An investigation into upheaval buckling of buried pipelines-I. Experimental apparatus and some observations. International Journal of Mechanical Sciences. 1995;37:943-63.

[11] Maltby TC, Calladine CR. An investigation into upheaval buckling of buried pipelines-II. Theory and analysis of experimental observations. International Journal of Mechanical Sciences. 1995;37:965-83.

[12] Liu R, Xiong H, Wu X, Yan S. Numerical studies on global buckling of subsea pipelines. Ocean Eng. 2014;78:62-72.

[13] Wang Z, Tang Y, Zhou L, Zhao Z, Wang C. Analytical solution for controlled lateral buckling of unburied subsea pipelines. Ocean Eng. 2017;146:140-50.

[14] Wang Z, Tang Y, Wang C. Analytical solution for lateral buckling of unburied subsea pipelines with distributed buoyancy section. Ocean Eng. 2017;146:115-24. 
[15] Wang Z, Tang Y, van der Heijden GHM. Analytical study of lateral thermal buckling for subsea pipelines with sleeper. ThinWalled Structures. 2018;122:17-29.

[16] Wang Z, Tang Y, van der Heijden GHM. Analytical study of distributed buoyancy sections to control lateral thermal buckling of subsea pipelines. Marine Structures. 2018;58:199-222.

[17] Wang Z, van der Heijden GHM, Tang Y. Analytical study of third-mode lateral thermal buckling for unburied subsea pipelines with sleeper. Engineering Structures. 2018;168:447-61.

[18] Zeng X, Duan M. Mode localization in lateral buckling of partially embedded submarine pipelines. International Journal of Solids and Structures. 2014;51:1991-9.

[19] Wang Z, van der Heijden GHM. Localised lateral buckling of partially embedded subsea pipelines with nonlinear soil resistance. Thin-Walled Structures. 2017;120:408-20.

[20] Wang Z, van der Heijden GHM, Tang Y. Localised upheaval buckling of buried subsea pipelines. Marine Structures. 2018;60:165-85.

[21] Taylor N, Gan AB. Submarine pipeline buckling-imperfection studies. Thin-Walled Structures. 1986;4:295-323.

[22] Taylor N, Tran V. Prop-imperfection subsea pipeline buckling. Marine Structures. 1993;6:325-58.

[23] Pedersen PT, Jensen JJ. Upheaval creep of buried heated pipelines with initial imperfections. Marine Structures. 1988;1:1122.

[24] Ballet JP, Hobbs RE. Asymmetric effects of prop imperfections on the upheaval buckling of pipelines. Thin-Walled Structures. 1992;13:355-73.

[25] Croll JGA. A simplified model of upheaval thermal buckling of subsea pipelines. Thin-Walled Structures. 1997;29:59-78.

[26] Karampour H, Albermani F, Gross J. On lateral and upheaval buckling of subsea pipelines. Engineering Structures. 2013;52:317-30.

[27] Karampour H, Albermani F. Experimental and numerical investigations of buckle interaction in subsea pipelines. Engineering Structures. 2014;66:81-8.

[28] Karampour H, Albermani F, Veidt M. Buckle interaction in deep subsea pipelines. Thin-Walled Structures. 2013;72:113-20.

[29] Shi R, Wang L, Guo Z, Yuan F. Upheaval buckling of a pipeline with prop imperfection on a plastic soft seabed. Thin-Walled Structures. 2013;65:1-6.

[30] Hong Z, Liu R, Liu W, Yan S. Study on lateral buckling characteristics of a submarine pipeline with a single arch symmetric initial imperfection. Ocean Eng. 2015;108:21-32.

[31] Wang Y, Zhang X, Zhao Y, Chen H, Duan M, Estefen SF. Perturbation analysis for upheaval buckling of imperfect buried pipelines based on nonlinear pipe-soil interaction. Ocean Eng. 2017;132:92-100.

[32] Liu R, Basu P, Xiong H. Laboratory tests and thermal buckling analysis for pipes buried in Bohai soft clay. Marine Structures. 2015;43:44-60.

[33] Wang Z, Chen Z, Liu H. Numerical study on upheaval buckling of pipe-in-pipe systems with full contact imperfections. Engineering Structures. 2015;99:264-71.

[34] Wang Z, Chen Z, Liu H, Zhang Z. Numerical study on lateral buckling of pipelines with imperfection and sleeper. Applied Ocean Research. 2017;68:103-13.

[35] Xu L, Lin M. Numerical study on critical axial forces of upheaval buckling for initially stressed submarine pipelines on uneven seabed. Ocean Eng. 2017;145:344-58.

[36] Zhang X, Duan M. Prediction of the upheaval buckling critical force for imperfect submarine pipelines. Ocean Eng. 2015;109:330-43.

[37] Zhang X, Soares CG, An C, Duan M. An unified formula for the critical force of lateral buckling of imperfect submarine pipelines. Ocean Eng. 2018;166:324-35.

[38] Liu R, Li C. Determinate dimension of numerical simulation model in submarine pipeline global buckling analysis. Ocean Eng. 2018;152:26-35.

[39] Hunt GW, Blackmore A. Homoclinic and heteroclinic solutions of upheaval buckling. Proceedings of the Royal Society of 
London A: Mathematical, Physical \& Engineering Sciences. 1997;355:2185-95. 